\title{
1 Involvement of translocon complex in hemoglobin import from infected 2 erythrocyte cytoplasm into the Plasmodium parasite
}

3 Priya Gupta ${ }^{1}$, Rajan Pandey ${ }^{2}$, Vandana Thakur ${ }^{3}$, Sadaf Parveen ${ }^{1}$, Inderjeet Kaur ${ }^{1}$, Ashutosh

4 Panda $^{1}$, Rashmita Bishi ${ }^{1}$, Sonali Mehrotra ${ }^{1}$, Dinesh Gupta $^{2}$, Asif Mohmmed ${ }^{3}$, Pawan

5 Malhotra $^{{ }^{*}}$

$6{ }^{1}$ Malaria Biology Group, International Centre for Genetic Engineering and Biotechnology,

7 Aruna Asaf Ali Marg, New Delhi-110067, India

$8{ }^{2}$ Translational Bioinformatics Group, International Centre for Genetic Engineering and

9 Biotechnology, Aruna Asaf Ali Marg, New Delhi-110067, India

${ }^{3}$ Parasite Cell Biology Group, International Centre for Genetic Engineering and

11 Biotechnology, Aruna Asaf Ali Marg, New Delhi-110067, India

12 * To whom correspondence should be addressed.

13 E-mail: pawanm@icgeb.res.in, pawanmal@gmail.com 


\section{Abstract}

Haemoglobin degradation is crucial for the growth and survival of Plasmodium falciparum in human erythrocytes. Although the process of $\mathrm{Hb}$ degradation has been studied in great detail, the mechanisms of $\mathrm{Hb}$ uptake remain ambiguous to date. Here, we characterized Heme Detoxification Protein (PfHDP), a protein localized in the parasitophorous vacuole, parasite food vacuole and infected erythrocyte cytosol for its role in $\mathrm{Hb}$ uptake. Immunoprecipitation of PfHDP-GFP fusion protein from a transgenic line using anti-GFP antibody and of Plasmodium parasite extract using anti-human $\mathrm{Hb}$ antibodies respectively, showed the association of $P f \mathrm{HDP} / \mathrm{Hb}$ with each other as well as with the members of PTEX translocon complex. Some of these associations such as $P f H D P / H b$ and $P f H D P / P f e x p-2$ interactions were confirmed by in vitro protein-protein interaction tools. To know the roles of PfHDP and translocon complex in $\mathrm{Hb}$ import into the parasites, we next studied the Hb uptake by the parasite in PfHDP knock-down line using the GlmS ribozyme strategy. PfHDP knock-down significantly reduced the $\mathrm{Hb}$ uptake in these parasites in comparison to the wild type parasites. Further, the transient knock-down of one of the members of the translocon complex; PfHSP101 showed considerable reduction in Hb uptake. Morphological analysis of PfHDP-HA-GlmS transgenic parasites in the presence of GlcN showed food vacuole abnormalities and parasite stress, thereby causing a growth defect in the development of these parasites. Together, we implicate the translocon complex in the trafficking of $\mathrm{PfHDP} / \mathrm{Hb}$ complex in the parasite and suggest a role for PfHDP in the uptake of $\mathrm{Hb}$ and parasite development. The study thus reveals new insights into the function of PfHDP, making it an extremely important target for developing new antimalarials. 


\section{Introduction}

Plasmodium falciparum is a leading cause of malaria with 219 million malaria cases and 4,35,000 deaths reported worldwide in the year 2018 (Organization 2019). Although drug therapies and vector control mechanisms for the containment of the disease have been developed, eradication of malaria has not been achieved yet (Rathore, McCutchan et al. 2005). The spread of resistance against the available antimalarials and unavailability of a highly efficacious vaccine poses a greater challenge to eradicate this dreaded disease. Early resistance against the artemisinin combination therapies, a present-day front-line therapy, had been reported in western Cambodia, later spreading across an expanding area of the Greater Mekong subregion (Woodrow and White 2017). Hence, there is an urgent need to identify new drug targets and molecules that can be targeted to develop effective vaccines to prevent the disease spread.

During the asexual stage of life cycle inside the human erythrocyte, the parasite digests $\mathrm{Hb}$ in a specialized organelle, the food vacuole. $\mathrm{Hb}$ is digested by the sequential action of a complex of proteases including plasmepsins, falcipains and falcilysin inside the food vacuole (Luker, Francis et al. 1996, Francis, Banerjee et al. 1997, Liu, Gluzman et al. 2005, Singh, Sijwali et al. 2006). These proteases cleave $\mathrm{Hb}$ to short peptides and finally to amino acids (Dalal and Klemba 2007). The free toxic by-product, heme, generated during the process is subsequently converted into an inert insoluble polymer; hemozoin (Ashong, Blench et al. 1989, Egan, Combrinck et al. 2002, Egan 2008). PfHDP,(Heme Detoxification Protein) has been shown to be extremely potent in converting heme to hemozoin (Jani, Nagarkatti et al. 2008). PfHDP, a food vacuole associated protein, possesses two heme binding sites and a $\mathrm{Hb}$ binding site (Gupta, Mehrotra et al. 2017) and is a part of a 200 kDa complex with other proteins including falcipain 2/2, Plasmepsin II, Plasmepsin IV, and histo-aspartic protease inside the food vacuole (Chugh, Sundararaman et al. 2013).

Although the mechanism of $\mathrm{Hb}$ degradation has been extensively studied; the mechanisms of uptake of the $\mathrm{Hb}$ from infected erythrocyte cytosol to the parasite remains poorly understood. Four distinct pathways have been proposed to assist in the uptake of $\mathrm{Hb}$ (Elliott, McIntosh et al. 2008, Lazarus, Schneider et al. 2008). The uptake begins with the folding of parasites around erythrocyte cytoplasm followed by the development of vesicles and cytostomes that continue uptake of $\mathrm{Hb}$ inside the parasite. After this step, phagosomes appear, which assist in the trafficking of $\mathrm{Hb}$. Finally, cytostomal invaginations elongate to form tubes that connect to 
the digestive vacuole at one end and to the parasite surface at the other end, opening to the erythrocyte cytosol. Despite all the microscopic evidence available, the molecules and adaptors that participate in delivering $\mathrm{Hb}$ to the food vacuole hold ambiguity. Based on immuno-electron microscopy, PfHDP was shown to be present in the vesicles that traffic $\mathrm{Hb}$ from the erythrocyte to the food vacuole of the parasite (Jani, Nagarkatti et al. 2008). PfHDP lacks a classical N-terminal signal sequence or PEXEL motif, which usually assists in the sorting and transporting of any protein to a destined site using the translocon complex. The translocon complex consisting of PTEX150, Exportin 2, PTEX88, HSP101 and Trx2 is used by the parasite to export proteins from the parasite to the erythrocyte cytoplasm (de KoningWard, Gilson et al. 2009).. Interaction of PfHDP and Hb paved the way for a hypothesis suggesting that PfHDP might be playing some role in the uptake of $\mathrm{Hb}$ from the erythrocyte cytoplasm (Gupta, Mehrotra et al. 2017).

In this study, we attempt to unravel one of the pathways involved in the uptake of $\mathrm{Hb}$ from the erythrocyte cytosol. Downregulation of PfHDP in the PfHDP-HAGlmS transgenic parasites led the parasites to take up less $\mathrm{Hb}$ from the erythrocyte and induced parasite stress. Immunoprecipitation of parasite cell lysates using anti-Hb and anti- GFP antibodies showed an association of PfHDP with $\mathrm{Hb}$ and with the components of the translocon complex, such as exportin-2, PTEX150 and HSP101. In silico and in vitro protein-protein interaction studies confirmed the association of PfHDP with Pfexp-2. Hence, we propose that PfHDP participates in the uptake of $\mathrm{Hb}$ from erythrocyte using the translocon complex. Functional insights into the role of PfHDP can help us design better inhibitors targeting both the heme and $\mathrm{Hb}$ binding PfHDP domains.

\section{Methods}

\section{Maintenance of $\boldsymbol{P}$. falciparum cultures and transfection}

The P. falciparum parasite line 3D7 was maintained as described previously (Trager and Jensen 1976). To generate a GFP overexpressing transfection vector construct, the entire open reading frame of PfHDP was amplified using HDPGFP-FP 5'GCAGATCTTTTTTCATCAGTATGAAAAAT AGATTTTATTAT 3' and HDPGFPRP 5'GC CCTAGGAAAAATGATGGGCTTATCTACTATAT3' primer set and cloned into the pSSPF2 vector to create a C terminal PfHDP-GFP fusion protein under the control of hsp86 promoter. P. falciparum 3D7 ring-stage parasites were transfected with $100 \mu \mathrm{g}$ of plasmid DNA by electroporation $(310 \mathrm{~V}, 950 \mu \mathrm{F})$ and the transfected parasites were selected using $2.5 \mathrm{nM}$ blasticidin (Crabb, Rug et al. 2004). Expression of the PfHDP-GFP fusion 
protein in transgenic $P$. falciparum blood-stage parasites was examined by western blotting and immunofluorescence. Protein bands were visualized using an ECL kit (Thermo Scientific, USA).

For transfection of knock-down constructs, C-terminal region of PfHDP was amplified using gene-specific primers, HDP GlmSHA FP 5'GCAGATCTTTGAACATAAGCCTGTAAAAAGGA C 3' and HDP GlmSHA RP 5' GCCTGCAGAAA AATGATGGGCTTATCTACTAT3' and cloned into the transfection vector pHA-glmS using PstI and BglII restriction sites to create a fusion of desired gene of interest (GOI) with HA-glmS at the 3' UTR under the control of native promoter. The ringstage parasites were transfected as mentioned and transgenic parasites were selected on alternate WR22910 drug ON and OFF cycles to ensure genomic integration of PfGOI-HAglmS constructs. The transgenic parasites were then subjected to clonal selection by serial dilution to obtain parasite line from a single genome integrated clone. The integration was checked by PCR amplification of genomic fragments using different sets of primers: (1) $(\mathrm{a} / \mathrm{b})$ HDP GlmSHA FP/ HDP GlmSHA RP (2) (c/d) HDPint check 5' GTAGAATGTATTTTTCATCAGT3'/ HAintcheck 5’TACGGATACGCATAATCGG3'.

\section{Cloning, Recombinant Expression, and Purification of exportin 2 protein}

The forward and reverse primers used for the cloning of C-terminal Plasmodium falciparum exportin 2 (Pfexp-2) were, 5' CCCCATGGGATCCATGAACAATTAAAGATATTTA 3' and 5' GCGCGGCCGCTTCTTTATTTTCATCTTTTTT 3', respectively. The PCR product of these primers was cloned into a pJET vector and subsequently subcloned into a pET28b vector. The gene cloned in the pET-28b vector was expressed in codon+ Escherichia coli cells and protein localized to inclusion bodies, which were isolated as described. Briefly, the inclusion bodies were solubilized in an $8 \mathrm{M}$ urea buffer (500 mM Tris, $150 \mathrm{mM} \mathrm{NaCl})$. The suspension was incubated for $1 \mathrm{~h}$ at room temperature (RT) and then centrifuged at 12,000 $\times g$ for $30 \mathrm{~min}$ at RT. The supernatant containing solubilized protein was kept for binding with $\mathrm{Ni}-\mathrm{NTA}^{+}$resin overnight at RT with constant shaking. After binding, the suspension was packed in a purification column, and flow-through was collected. The resin was washed with an $8 \mathrm{M}$ urea buffer containing $10 \mathrm{mM}$ imidazole. Bound protein(s) was eluted in an $8 \mathrm{M}$ urea buffer containing different concentrations of imidazole. Eluted protein fractions were analyzed by $12 \%$ SDS-PAGE. The eluted fractions containing the purified protein, were pooled and concentrated. The refolding method was adopted from a standard universal protocol (Tsumoto, Ejima et al. 2003). The protein was refolded gradually by decreasing the 
urea concentration $(6 \mathrm{M}, 4 \mathrm{M}, 2 \mathrm{M}, 1 \mathrm{M}, 0 \mathrm{M})$ in the refolding buffer $(0.05 \mathrm{M}$ Tris, $\mathrm{pH} 8,1 \mathrm{mM}$ EDTA, 0.5 M arginine, $0.4 \mathrm{mM}$ Triton X-100, $1 \mathrm{mM}$ reduced glutathione, $0.5 \mathrm{mM}$ oxidized glutathione). The refolded proteins were concentrated and dialyzed against $0.05 \mathrm{M}$ Tris, $\mathrm{pH}$ 8 , and $0.15 \mathrm{M} \mathrm{NaCl}$ and stored at $-80^{\circ} \mathrm{C}$. The purified protein was analyzed on a $12 \% \mathrm{SDS}$ polyacrylamide gel followed by western blotting with anti His HRP antibody.

\section{Generation of antibodies against recombinant PfHDP and Pfexp-2}

Antibodies against recombinant $P f \mathrm{HDP}$ and $P f \exp -2$ were raised in mice and rabbit. For this 5-6-week-old female BALB/c mice were immunized with $25 \mu \mathrm{g}$ of recombinant HDP protein emulsified in Freund's complete adjuvant on day 0 followed by three boosts of proteins emulsified with Freund's incomplete adjuvant on days 14, 28 and 42. The animals were bled for serum collection on day 49. In case of rabbits, NewZealand white female rabbits were immunized with $200 \mu \mathrm{g}$ of recombinant PfHDP and Pfexp-2, respectively, emulsified in Freund's complete adjuvant on day 0 followed by three boosts emulsified with Freund's incomplete adjuvant on days 21, 42 and 63. The animals were bled for serum collection on day 70. The antibody titer in serum samples were quantified by enzyme-linked immunosorbent assay (ELISA).

\section{In vitro protein-protein interaction analysis}

ELISA based protein-protein interaction analysis was performed as described previously (Paul, Deshmukh et al. 2017). Briefly, a 96-well microtiter plate was coated overnight at $4^{\circ} \mathrm{C}$ with $50 \mathrm{ng}$ recombinant PfHDP protein. Another unrelated Plasmodium falciparum recombinant protein, Ddi was coated as negative control. After blocking the wells with 5\% milk in PBS for $2 \mathrm{~h}$, recombinant $P$ fexp-2 was added in different amounts ranging from 0 to $100 \mathrm{ng}$, and the plate incubated for $3 \mathrm{~h}$ at $37^{\circ} \mathrm{C}$. The interaction was detected using antibodies against exportin 2 (1:500). Incubation with HRP conjugated anti-rabbit antibodies (1:3000) was done for $1 \mathrm{~h}$ and quantified after adding the substrate OPD by measuring the resulting absorbance at $490 \mathrm{~nm}$.

Far western assays were performed according to the protocol described earlier (Wu, Li et al. 2007). Briefly 1-5 $\mu \mathrm{g}$ of recombinant PfHDP and an unrelated Plasmodium protein PfMLH/MBP were separated by SDS-PAGE and transferred to a nitrocellulose membrane. The proteins were first denatured and then renatured on the membrane itself. The membranes were blocked with $5 \%$ skimmed milk and incubated with $2 \mu \mathrm{g} / \mathrm{mL}$ of purified interacting bait 
proteins i.e, $\mathrm{Hb}$ and exp 2 in protein binding buffer $(100 \mathrm{mM} \mathrm{NaCl}, 20 \mathrm{mM}$ Tris (pH 7.6), 0.5 mM EDTA, $10 \%$ glycerol, and $1 \mathrm{mM}$ DTT) for $2 \mathrm{~h}$ at room temp. After washing the nonspecific proteins, membranes were incubated with rabbit anti- $\mathrm{Hb} /$ anti-exp-2 followed by incubation with HRP conjugated anti-rabbit antibodies (1:100000) for $1 \mathrm{~h}$ at RT. Finally, membranes were imaged with a Biorad ECL chemidoc.

For in vitro co-immunoprecipitation, $1 \mu \mathrm{g}$ of each protein PfHDP and $\mathrm{Hb}$ were incubated together at room temperature for $2 \mathrm{~h}$ in a reaction volume of $100 \mu \mathrm{L}$ containing $1 \times$ binding buffer $(50 \mathrm{mM}$ phosphate buffer at $\mathrm{pH} 7.0,75 \mathrm{mM} \mathrm{NaCl}, 2.5 \mathrm{mM}$ EDTA at $\mathrm{pH} 8.0$, and 5 $\mathrm{mM} \mathrm{MgCl}_{2}$ ), $0.1 \%$ Nonidet P 40, and $10 \mathrm{mM}$ DTT. The reaction mix was incubated for $2 \mathrm{~h}$ at $4^{\circ} \mathrm{C}$ with $20 \mu \mathrm{L}$ of pre-equilibrated Protein A/G conjugated antibody beads. The beads were centrifuged at $1,000 \times \mathrm{g}$ for $5 \mathrm{~min}$, washed with $200 \mu \mathrm{L}$ of binding buffer containing $400 \mathrm{mM}$ $\mathrm{NaCl}$, boiled for $5 \mathrm{~min}$ in SDS/PAGE reducing loading buffer, electrophoresed, immunoblotted, and probed. Protein A/G beads conjugated to preimmune sera were used as a negative control.

The Surface Plasmon Resonance analysis was carried out on the Biacore T200 instrument (GE Healthcare). Over 9500 Response Units of the recombinant HDP protein were immobilized on S-Series CM5 sensor chip (GE Healthcare) using 10mM sodium acetate pH4.0 solution (GE Healthcare). The surface of the sensor chip was blocked with $1 \mathrm{M}$ ethanolamine- $\mathrm{HCl}$ pH8.5 (GE Healthcare). Recombinant $P$ fexp-2/Hb at increasing concentrations was injected over the immobilized HDP and on the reference flow cell at a flow rate of $20 \mu \mathrm{min}^{-1}$. The kinetic parameters of the interaction and binding responses in the steady-state region of the sensogram were analyzed using Biacore evaluation software, version 4.1.1 (GE Healthcare).

\section{Indirect immunofluorescence assay}

Briefly, thin smears of parasite cultures were made on a glass slide and fixed with a mixture of methanol/acetone. Slides were blocked in blocking buffer (PBS, 3\% BSA) for $2 \mathrm{~h}$ at $37{ }^{\circ} \mathrm{C}$. Immunostaining was performed using primary antibodies (anti-PfHDP antibody 1:100, anti$\mathrm{Hb}$ antibody 1:100, anti-Pfexp-2 antibody 1:50, anti-PfPTEX150 antibody 1:50) and appropriate secondary antibody Alexa flour 594 goat anti-mice (1:500) and Alexa flour 488 goat anti-rabbit (1:500). For liquid staining the parasite samples were fixed in $4 \%$ paraformaldehyde/ glutaraldehyde. The fixed samples were permeabilized using $0.1 \%$ triton 
X100. The cells were blocked in $10 \%$ FBS for $2 \mathrm{~h}$ at RT. Immunostaining was then performed using primary antibodies overnight at $4{ }^{\circ} \mathrm{C}$. Appropriate secondary antibody, Alexa flour 594 goat anti-mice (1:500) and Alexa flour 488 goat anti-rabbit (1:500) was then added to stain the parasites for $1 \mathrm{~h}$ at RT. The nucleus of the parasites was stained using DAPI. For imaging, a drop of the suspension was taken on a slide and viewed under the microscope.

The transgenic parasite suspension was incubated with DAPI $(2 \mathrm{ng} / \mathrm{ml})$ in PBS at RT for $10 \mathrm{~min}$ and parasites were observed under a microscope to visualize the GFP expression. The images were captured using a Nikon A1 Confocal Microscope and exported as 8-bit RGB files. Images were analyzed using Nikon NIS Elements v 4.0software. Imaris image was created using the software IMARIS v 4.

\section{Immunoprecipitation reaction}

Immunoprecipitation experiments were performed using the Pierce Crosslink Immunoprecipitation Kit (Product \#26147). Briefly, synchronized Plasmodium falciparum 3D7 mid-late trophozoites were enriched from the uninfected population using density-based percoll treatment. The parasite pellet obtained was treated with Streptolysin O to lyse the erythrocyte membrane. The pellet containing the parasite surrounded by the parasitophorous vacuolar membrane was washed with PBS until lysis of RBC stopped by centrifugation at $15000 \times \mathrm{g}$ for $1 \mathrm{~min}$ and the pellet was then resuspended in PBS. The parasite pellet was then lysed using the RIPA buffer (250mM Tris, $150 \mathrm{mM} \mathrm{NaCl,} \mathrm{1mM} \mathrm{EDTA,} 1 \%$ NP-40, 5\% glycerol: pH 7.4) containing protease and phosphatase inhibitor cocktails (Roche) for $30 \mathrm{~min}$ at $4{ }^{\circ} \mathrm{C}$ with intermittent mixing. Lysate was clarified by centrifugation at $15000 \times \mathrm{g}$ for 30 min. The supernatant protein concentration was determined by the BCA Protein estimation assay kit (Pierce) using BSA standards as reference. Approximately $1 \mathrm{mg}$ of total protein was incubated with about $10 \mu \mathrm{g}$ of anti- $\mathrm{Hb}$ antibody, cross-linked to $10 \mu \mathrm{l}$ of Protein A agarose beads using disuccinimidyl suberate (DSS) as crosslinker, for $12 \mathrm{~h}$ at $4^{\circ} \mathrm{C}$ with constant mixing. An equal amount of protein was allowed to bind to beads conjugated to the preimmune antibody as a control. Following binding, beads were washed with the wash Buffer and the bound proteins were eluted from the beads using the Elution Buffer (TrisGlycine $\mathrm{pH}$ 2.8). Proteins in the immunoprecipitated samples were digested by in-solution trypsin digestion. Samples were brought to a final volume of $100 \mu \mathrm{l}$ in $50 \mathrm{mM}$ Ammonium Bicarbonate (Sigma, U.S.A.) buffer to adjust the $\mathrm{pH}$ to 7.8 , reduced with $10 \mathrm{mM}$ DTT for $1 \mathrm{~h}$ at RT followed by alkylation with $40 \mathrm{mM}$ Iodoacetamide (Sigma, U.S.A.) for $1 \mathrm{~h}$ at RT under 
dark conditions. Proteins were digested by the addition of Promega sequencing grade modified trypsin (V511A) at a ratio 1:50 (w/w) of trypsin: protein. For complete digestion, samples were placed in a water-bath at $37^{\circ} \mathrm{C}$ for $16 \mathrm{~h}$. After digestion, extracted peptides were acidified with $0.1 \%$ formic Acid and analyzed by mass spectrometry.

The SLO-treated trophozoite stage lysate of PfHDP-GFP transgenic parasites was immunoprecipitated using GFP-Trap ${ }^{\circledR}$ A K Kit (Chromotek)/ anti-GFP antibody following the manufacturer's instructions. GFP-Trap ${ }^{\circledR}$ A A beads/ anti-GFP antibody was allowed to bind to parasite lysate by tumbling the tube end-over-end. Proteins were eluted in a $50 \mu l$ elution buffer, digested with trypsin and peptides were analysed by mass spectrometry. 3D7 parasites treated using the same protocol was used as negative control.

\section{Conditional knock down assay}

The functional role of PfHDP was determined by knocking down the HDP mRNA with glucosamine. Effect of knock down on parasite invasion was evaluated with 3D7 strain of $P$. falciparum as the control. The parasite lines (PfHDP-HA-GlmS transgenic and 3D7) were synchronized using 5\% sorbitol and the growth assay was set at the mid ring stage with a haematocrit and parasitemia of synchronized ring stage culture adjusted to $2 \%$ and $1 \%$, respectively. Glucosamine was added to the parasite culture at varying concentrations $(0$, $1.25 \mathrm{mM}, 2.5 \mathrm{mM}$, and $5 \mathrm{mM}$ ). Parasite growth was monitored microscopically by Giemsastained smears. The parasitemia was estimated after an incubation of $40 \mathrm{~h}$ in the next cycle and also in the second cycle using flow cytometry. Briefly, cells from samples were pelleted and washed with PBS followed by staining with ethidium bromide $(10 \mu \mathrm{g} / \mathrm{ml})$ for $20 \mathrm{~min}$ at $37^{\circ} \mathrm{C}$ in the dark. The cells were subsequently washed twice with PBS and analyzed on FACS calibur (Becton Dickinson) using the Cell Quest software. Fluorescence signal (FL2) was detected with the 590nm band pass filter using an excitation laser of $488 \mathrm{~nm}$ collecting 100000 cells per sample. Uninfected RBCs stained in similar manner were used as control. Following acquisition, data were analyzed for percentage parasitemia of each sample by determining the proportion of FL2-positive cells using Cell Quest.

\section{Protein-protein docking analysis}

PlasmoDB release (release 48) was used to retrieve sequences of Pfexp-2 and PfHDP proteins (Bahl, Brunk et al. 2002). The predicted 3D model of PfHDP was used as reported previously (Gupta, Mehrotra et al. 2017). The cryo-EM structure of Pfexp-2 has been 
resolved (Ho, Beck et al. 2018), hence its corresponding PDB structure (6E10.pdb) was retrieved from the RCSB PDB. The protein-protein docking was performed using PatchDock based on shape complementarity principles (Schneidman-Duhovny, Inbar et al. 2005). Energy refinement was performed for top 100 docked conformations using FireDock (Mashiach, Schneidman-Duhovny et al. 2008). Different conformations showing global energy $\leq-5.0 \mathrm{kcal} / \mathrm{mol}$ for $P f$ exp-2-PfHDP complexes were further analysed. PyMol was used to visualize protein complexes and generate images (https://pymol.org/2/). DIMPLOT was used to retrieve and visualize residues within $4 \AA$ of interacting docked Pfexp- 2-PfHDP complexes (Laskowski and Swindells 2011). Protein Interactions Calculator (PIC) was used to identify $P f$ exp-2-PfHDP interactions which recognizes various kinds of interactions, such as disulphide bonds, hydrophobic interactions, ionic interactions, hydrogen bonds, aromaticaromatic interactions, aromatic-sulphur interactions, and cation $-\pi$ interactions within a protein or between proteins in a complex (Tina, Bhadra et al. 2007).

\section{Results}

\section{$P f H D P$ interacts with $\mathrm{Hb}$ as well with the members of Plasmodium translocon complex}

To identify the protein(s) associated with $P f \mathrm{HDP}$, we generated a parasite line expressing Cterminal GFP-tagged HDP in Plasmodium falciparum. PfHDP-GFP was expressed in an episomal construct; pSSPF2 vector (Sup Fig. 1A-D) with GFP tag at 3' end under the control of HSP86 promoter. Western blot analysis using anti- GFP antibodies recognised two bands in PfHDP-GFP in transgenic parasite lysates, one corresponding to PfHDP-GFP $\sim 50 \mathrm{kDa}$ and the other at $\sim 25 \mathrm{kDa}$. The lower band could be a result of processing of HDP-GFP in the lysate samples (Sup Fig. 1E). Indirect immunofluorescence assay of transgenic PfHDP-GFP asexual blood stages showed that $P f H D P$ is localized in the vesicles transported to the food vacuole (Fig. 1A). Co-staining of these transgenic parasites with BODIPY ceramide stain followed by live cell imaging revealed that PfHDP-GFP vesicles are present at the parasitophorous vacuole membrane as well as near the food vacuole indicating that $P f \mathrm{HDP}$ is trafficked to the food vacuole as well as to the erythrocyte cytoplasm (Fig. 1B). To ascertain whether $P f \mathrm{HDP}-\mathrm{GFP}$ was trafficked to the erythrocyte cytosol, we fractionated the transgenic parasites using streptolysin $\mathrm{O}$ to separate the parasite and erythrocyte cytoplasm fractions. Western blot analysis of both fractions using anti-GFP antiserum recognized bands at 
$\sim 50 \mathrm{kDa}$ and $\sim 25 \mathrm{kDa}$ in parasite cytoplasm and a band at $\sim 25 \mathrm{kDa}$ in erythrocyte cytoplasm (Sup Fig. 1F). Together, these results demonstrated that PfHDP-GFP was trafficked to the erythrocyte cytosol besides being transported to the food vacuole for the hemozoin formation. Western blot analysis of Streptolysin $O$ treated fractions of infected erythrocytes and immunofluorescence assays using anti-PfHDP antibodies further validated that PfHDP is present both inside the parasite as well as in the erythrocyte cytoplasm (Sup Fig. 2A-B).

We next examined the PfHDP-GFP interactome in the PfHDP-GFP parasite line using a GFP pull-down assay. Briefly, PfHDP-GFP protein was pulled down from cell lysates together with interacting partners, if any, using GFP-Trap beads bound with GFP antisera. Bound and eluted proteins were digested with trypsin and the released peptides were analysed by mass spectrometry to identify the interacting partners. In addition to the food vacuole proteases like Plasmepsin and falcipain 2, which are already shown to be a part of hemozoin formation complex (Chugh, Sundararaman et al. 2013), we found several proteins in the pull-down results including $\mathrm{Hb}$ as an interacting protein of PfHDP. Additionally, members of the translocon complex including Pfexp-2, PTEX150, PTEX88 were identified in the immunoprecipitants (Table1). None of these proteins were pulled down from the lysates of $P$. falciparum 3D7, which served as a negative control. Together, these results suggested an association of $P f \mathrm{HDP}$ with $\mathrm{Hb}$ as well as with the components of the translocon complex. To confirm the involvement of translocon complex in the trafficking of PfHDP-Hb complex inside the infected asexual blood stage parasites, immunoprecipitation of Streptolysin O clarified trophozoite stage parasite extract was performed using anti-human $\mathrm{Hb}$ antibody with 3D7 lysate and immunoprecipitate was subjected to mass spectrometric analysis. PfHDP protein was identified as an interacting partner of $\mathrm{Hb}$ inside the parasite along with Exp-2, PTEX150, PTEX88, HSP101 and Trx2 proteins (Table 2). None of these proteins were detected in the immunoprecipitate with pre-immune serum.

To provide further evidence(s) for the association between $\mathrm{Hb}$ and PfHDP, co-localization and in vitro protein-protein interaction studies such as co-immunoprecipitation and farwestern analysis were performed. For the far-western analysis, recombinant HDP protein that served as a bait was resolved on the SDS-PAGE gel and $\mathrm{Hb}$ was allowed to interact with bait protein on the nitrocellulose membrane, bound protein was probed with anti-Hb antibody. A high affinity interaction between PfHDP and $\mathrm{Hb}$ was observed (Sup Fig. 3A). Secondly, we performed interaction studies between PfHDP and $\mathrm{Hb}$ and co-immunoprecipitation analysis was carried out using anti- HDP antiserum. As seen in Sup Fig. 3B, anti-HDP antisera could 
pull down $\mathrm{Hb}$ from a mixture of the two proteins as analyzed by western blot analysis using anti-human $\mathrm{Hb}$ antibody. Recombinant ClpQ protein, a mitochondrial protein, was used as a negative control. Immunolocalization studies were next performed to localize PfHDP and human $\mathrm{Hb}$ protein together in trophozoite stage parasites using anti-PfHDP and anti-Hb antibodies. PfHDP co-localized considerably with human $\mathrm{Hb}$ with a Pearson coefficient of 0.81 , both inside the parasite as well as in the erythrocyte cytosol (Fig. 1C). We further analysed the kinetics of the binding of PfHDP to Hb using the Surface Plasmon Resonance. The recombinant HDP protein was immobilized on a CM5 SPR chip and $\mathrm{Hb}$ was allowed to interact with the immobilized protein at different concentrations ranging from $31.125 \mu \mathrm{g} / \mathrm{ml}$ to $500 \mu \mathrm{g} / \mathrm{ml}$. The sensogram showed a dose dependent increase in binding of the $\mathrm{Hb}$ with time to the immobilized PfHDP protein with an equilibrium dissociation constant of $4.3^{*} 10^{-6} \mathrm{M}$ (Fig. 1D). The binding followed a two-state reaction suggesting more than one binding site for binding of $P f H D P$ to $\mathrm{Hb}$.

To validate the involvement of components of translocon complex in Hb/PfHDP trafficking if any, co-localization studies were performed by immune-staining trophozoite stage parasites with the respective antibodies. Immunofluorescence assay showed that $P f \mathrm{HDP}$ and $\mathrm{Hb}$ indeed co-localized with the two components of the translocon complex: Pfexp-2 and PfPTEX150, at the parasitophorous vacuolar membrane (Fig. 2A-B and 3A). The Pearson coefficient of the correlation was found to be above $\sim 0.6$. Together these results point towards a likely role of the translocon complex in the transport of PfHDP-Hb complex from erythrocyte cytoplasm into the parasite.

\section{PfHDP interacts with exportin 2 at the parasitophorous vacuolar membrane}

We next sought to analyse the interaction of PfHDP with one of the members of the translocon complex; Pfexp-2 that has been suggested to be a pore forming protein on the parasitophorous vacuolar membrane (Garten, Nasamu et al. 2018, Sanders, Dickerman et al. 2019). To study the interaction between PfHDP and Pfexp-2, we cloned and expressed a Cterminal fragment of Pfexp-2 encompassing amino acids 139-285 in E. coli (Sup Fig. 4A). The protein was purified to homogeneity using the Ni-NTA ${ }^{+}$column. The purified protein migrated at a molecular size of $\sim 20 \mathrm{kDa}$ as seen in Coomassie stained SDS-PAGE and western blot experiment performed using anti-His-HRP antibody (Sup Fig. 4B-C). Specific antibodies were generated against recombinant $P f \exp -2$ protein in rabbit. The antisera 
recognized a band of $\sim 33 \mathrm{kDa}$ in the $3 \mathrm{D} 7$ parasite cell lysate, which corresponds to the monomeric form of native Pfexp-2 (Sup Fig. 4D).

We further employed in vitro protein- protein interaction tools such as in vitro ELISA based protein binding assay and far-western binding analysis to assess PfHDP interaction with $P f$ exp-2. Recombinant $P f$ HDP protein interacted with $P f \exp -2$ in a concentration dependent manner in (Sup Fig. 4E). A nonspecific recombinant PfDdi protein was used as a negative control, which showed no significant interaction with Pfexp-2. For the far-western binding analysis we used recombinant PfHDP protein as a bait and $P f \exp -2$ was allowed to interact with bait protein on the nitrocellulose membrane and probed with anti-exp-2 antibody. A nonspecific purified MBP protein was used as a negative control. As evident in Fig. 3B, PfHDP interacts specifically with Pfexp-2 (Fig. 3B). To know whether PfHDP interacts with $P f$ exp-2 in the cell, parasite lysate from PfHDP-GFP transgenic line was immunoprecipitated using GFP-TRAP column and elutes from the GFP pull down assay were analysed by western blot analysis using anti-Pfexp-2 antibody. The parasite lysate from 3D7 parasite line was used as a negative control. As seen in Supplementary figure 4F, PfHDP-GFP fusion protein interacted with $P f$ exp-2, which was detected in the western blot analysis, whereas 3D7 lysate did not show any Pfexp-2 band. To study the kinetics of the binding of PfHDP to Pfexp-2, we performed the Surface Plasmon Resonance analysis by immobilizing the recombinant HDP protein on a CM5 chip using EDC-NHS coupling. Recombinant Pfexp-2 was allowed to interact with the immobilized protein at different concentrations ranging from $0.625 \mu \mathrm{M}$ to $10 \mu \mathrm{M}$. The sensogram showed a dose dependent increase in binding of the $P f$ exp-2 with time to the immobilized PfHDP, with an equilibrium dissociation constant of $1.1^{*} 10^{-6} \mathrm{M}$ (Fig. 3C). Together, these binding studies unequivocally suggested an interaction between PfHDP and Pfexp-2.

To identify the interaction sites for the Pfexp-2 and PfHDP, we carried out in silico interaction analysis using the already known structure of the malaria translocon complex, 6E10.pdb and HDP structure model. In silico docking analysis of Pfexp-2 monomer with PfHDP using PatchDock and energy refinement on top 100 conformations were carried out using default parameters. Next, the docked conformations, with global binding energy better than $-5 \mathrm{kcal} / \mathrm{mol}$ were analyzed. Interestingly, the docking analysis showed that PfHDP binds to the multiple sites of Pfexp-2, which includes Linker helix (L), globular domain body (B) and transmembrane domain (T) of Pfexp-2 (Fig. 3D) with highest binding energy observed for $P f H D P-P f$ exp-2 in the Linker region $(-39.57 \mathrm{kcal} / \mathrm{mol}$, pose 1). Similarly, the best binding 
score observed for PfHDP binding to the globular domain body (B) and transmembrane domain (T) of Pfexp-2 was -17.08 (pose 2) and $-6.35 \mathrm{kcal} / \mathrm{mol}$ (pose 3), respectively. The PPI analysis showed gradual changes in the PfHDP residues, which interacts with different pockets of Pfexp-2 (Supplementary Table 1) however PfHDP TYR56 and ASN59 residues binding remain consistent and were present within $4 \AA$ of $P f$ exp- 2 in almost all conformations analyzed for Pfexp-2-PfHDP complexes (Sup Fig. 5). Thus, our bioinformatics analysis further supported an interaction between $P f$ exp-2 and PfHDP.

\section{Knockdown of $P f H D P$ results in parasite stress and low levels of $\mathrm{Hb}$ inside the parasite}

To illustrate the functional importance of PfHDP protein in parasite biology in particularly for $\mathrm{Hb}$ import at asexual blood stages, a transgenic line with the genomic locus of PfHDP fused to GlmS ribozyme system was generated. The GlmS ribozyme is transcribed along with the gene. This inducible knockdown system uses glucosamine as an inducer. In the presence of glucosamine, which binds to ribozyme inducing the cleavage of the chimeric mRNA and hence knocking-down the respective targets (Sup Fig. 6A). The transgenic line was generated using pHA-GlmS vector-based constructs (Sup Fig. 6B-C). The integrants were selected using WR22910 selection, followed by clonal selection by limited dilution to obtain a pure conditional knockdown parasite line. The integration was confirmed by PCR amplification using different combinations of primer sets (Sup Fig. 6D). Expression of the HA tagged fusion protein and the native protein were confirmed by western blot analysis of the transgenic parasites using anti-HA antibody, which detected a single band at $\sim 50 \mathrm{kDa}$, corresponding to the size of dimeric native protein in the parasite (Sup Fig. 6E). No such band was detected in 3D7 parasite lysate was used as a negative control

To study the effect of the knockdown on the expression of PfHDP protein, the late trophozoite stages of transgenic parasites were treated with different concentrations of GlcN (0mM, 1.25mM, 2.5mM, respectively). The parasites were harvested in the next cycle at $42-$ 44 hpi and saponin treated parasites were lysed in RIPA buffer. Expression of the fusion protein was analysed by western blot analysis of the lysates from transgenic parasites using anti-HA antibody. A considerable reduction in PfHDP protein was seen in the presence of different concentrations of GlcN (Fig. 4A). PfBiP, a constitutively expressed endoplasmic reticulum chaperone protein, was used as a loading control. We next studied the effect of the knockdown of this protein on the growth of the parasites. GlcN was added at the ring stage parasites $16-20$ hpi at varying concentrations $(0 \mathrm{mM}, 1.25 \mathrm{mM}, 2.5 \mathrm{mM})$ and the growth was 
monitored till the formation of new rings up till two invasion cycles. In the first cycle, a slight decrease in the parasitemia was observed at $2.5 \mathrm{mM}$ GlcN concentration. However, when the parasites were allowed to progress to the second cycle, growth inhibition of $\sim 40 \%$ was observed in PfHDP-HAGlmS knockdown parasites (Fig. 4B). The inability of GlcN to inhibit more than $50 \%$ parasite growth could be attributed to the incomplete knockdown of the protein in the first cycle, as the synthesis of PfHDP begins as early as the ring stages of the parasite. Giemsa smears in the second cycle of growth demonstrated an induction of parasite stress and food vacuole abnormalities (Fig. 4C). The 3D7 parasites treated similarly with varied GlcN concentrations were used as a negative control. We further analysed the $\mathrm{Hb}$ levels in the knockdown parasites by western blotting using anti-Hb antibody. A considerable decrease in the amounts of $\mathrm{Hb}$ was seen inside the $\mathrm{GlcN}$ treated parasites (Fig. 4A). PfBiP was used as a loading control and it did not show any change in expression. We next studied the expression/localization of PfHDP and Plasmepsin 2 in the PfHDP knock-down parasites. Interestingly, we observed an inappropriate expression for Plasmepsin 2, a food vacuole protease in these stressed parasites (Fig. 4D). Overall, these results demonstrated a role of $P f H D P$ in $\mathrm{Hb}$ uptake and its impact on $\mathrm{Hz}$ formation.

\section{Parasites expressing low levels of PfHSP101 protein display low levels of $\mathrm{Hb}$ inside the parasite}

To characterize the role of components of the translocon complex in $\mathrm{Hb}$ trafficking, in particular the $\mathrm{Hb}$ import, we next studied the uptake of $\mathrm{Hb}$ in PfHSP101-DDDHA (Beck, Muralidharan et al. 2014)knockdown lines. For the same, the PfHSP101-DDD-HA parasites were grown in the presence of trimethoprim (TMP) (Fig. 5A); removal of TMP causes functional interference of PfHSP101 protein, thereby leading to its knockdown (Beck, Muralidharan et al. 2014). Briefly, the late trophozoite stages of parasites were treated with different concentrations of TMP $(0 \mu \mathrm{M}, 2.5 \mu \mathrm{M}, 5 \mu \mathrm{M}$, respectively) and these untreated $\mathrm{v} / \mathrm{s}$ treated parasites were harvested in the next cycle at 42-44 hpi. The saponin lysed parasites were subjected to lysis in RIPA buffer and $\mathrm{Hb}$ levels in the control v/s knockdown parasites were analysed by western blot analysis using anti-Hb antibody. A remarkable decrease in levels of $\mathrm{Hb}$ was observed in the TMP untreated parasites where PfHSP101 protein had been reduced considerably in comparison to the TMP treated parasites (Fig. 5B). PfBiP was used as a loading control. Reduction in PfHSP101 levels was confirmed in TMP untreated parasite lysates using anti- HA antibody to confirm the successful knockdown of HSP101 protein. 
In summary, these results advocated a role for PfHDP in trafficking/transport of $\mathrm{Hb}$ into the parasite. Based on the subcellular localization of PfHDP, its interaction with the $\mathrm{Hb}$ and with Pfexp-2, and its effect on the uptake of $\mathrm{Hb}$ in knockdown parasites, we propose a model suggesting that $P f \mathrm{HDP}$ interacts with $\mathrm{Hb}$ in infected erythrocyte. The PfHDP-Hb complex is then taken into the parasitophorous vacuole through the translocon complex by the interaction of $P f H D P$ with $P f$ exp-2. The $P f H D P-H b$ complex is subsequently taken up by the parasite and gets translocated to the food vacuole by vesicular trafficking.

\section{Discussion}

$\mathrm{Hb}$ uptake and its degradation are highly crucial processes for the growth of $P$. falciparum. The degradation of $\mathrm{Hb}$ results in the generation of amino acids that are utilized by the parasite for its protein synthesis. Heme generated as a by-product during the process of $\mathrm{Hb}$ catabolism is highly toxic for the survival of parasites. Heme Detoxification Protein has earlier been shown to be involved in the conversion of heme to an inert polymer hemozoin (Jani, Nagarkatti et al. 2008). In the present study, we aimed to understand Hb uptake/trafficking inside the infected parasites with a possible role of PfHDP and translocon complex in $\mathrm{Hb}$ inward trafficking.

It has been reported earlier that PfHDP is exported into the infected erythrocyte cytosol and takes a circuitous trafficking route to reach the food vacuole and catalyzes in $\mathrm{Hz}$ formation in the food vacuole (Jani, Nagarkatti et al. 2008). These authors further showed using antiPfHDP antibody as well as C- or N-tagged C-Myc-HDP protein that the trafficking of PfHDP to the cytosol of RBCs does not occur via the classical secretory pathway and the inbound HDP protein(s) are trafficked via their cytosomal uptake and vesicular trafficking (Jani, Nagarkatti et al. 2008). However, questions that remain unanswered is that how PfHDP is exported/imported into the erythrocyte cytosol or imported into the parasite back? To gain insights into the mode of trafficking of PfHDP into the infected parasites from RBC cytosol, a C- terminal GFP fusion HDP overexpressing parasite line under the control of Hsp86 promoter was generated. Confocal microscopy studies showed that the inbound PfHDP-GFP protein is trafficked via vesicular trafficking. SLO treatment on these transgenic parasites showed the expression of PfHDP-GFP fusion protein in the SLO soup suggesting that PfHDP-GFP protein is exported to PVM and to the erythrocyte cytoplasm. To further illustrate the import of $P f H D P$, immunoprecipitation analysis of PfHDP-GFP transgenic line or 3D7 line lysates with either GFP trap/anti GFP antibody or anti-Hb antibody were 
performed. Both the immunoprecipitates showed the presence of $P f H D P$ and $\mathrm{Hb}$ together along with the members of the translocon complex (de Koning-Ward, Gilson et al. 2009) such as PfPTEX150, PfExp-2 and PfHSP101, thereby implicating the role of PfHDP and translocon complex in $\mathrm{Hb}$ trafficking. Earlier, we have shown that PfHDP binds both heme as well as $\mathrm{Hb}$ (Gupta, Mehrotra et al. 2017). Based on these observations, we propose a model depicting PfHDP as an adapter protein that interacts with $\mathrm{Hb}$ in the erythrocyte cytoplasm and helps in the intake of $\mathrm{Hb}$ via the translocon complex.

To shed more lights into the proposed model, we expressed a C-terminal fragment of Pfexp-2 and raised specific antibodies against Pfexp-2. Additionally, an anti-peptide PTEX-150 antibody was generated. Both, PfHDP or $\mathrm{Hb}$ colocalized well with either Pfexp-2 or PfPTEX150 at the parasitophorous vacuolar membrane with a pearson's coefficient of $>0.5$, advocating a role for translocon complex in $\mathrm{Hb}$ import. A recent near atomic resolution cryoEM structure of endogenous translocon complex revealed that Pfexp-2 and PfPTEX150 intermingle to form a static, funnel shaped pseudo-sevenfold symmetric protein conducting channel spanning the vacuole membrane (Chi-Min Ho et al., 2018). To support further on the role of PfHDP protein in import of $\mathrm{Hb}$ from erythrocyte cytoplasm, interaction studies between $P f H D P$ and $\mathrm{Hb}$ or Pfexp-2 were performed by far western blot analysis or by Surface Plasma Resonance analysis. Recombinant HDP interacted with both $\mathrm{Hb}$ as well as $P f$ exp-2 with considerable affinities, thereby suggesting that PfHDP-Hb complex formed in infected erythrocyte cytoplasm is translocated through the static funnel by binding to Pfexp2. These results were also substantiated by docking studies between PfHDP and Pfexp-2

To study the functional relevance of PfHDP and components of translocon complex in parasite import of $\mathrm{Hb}$, a PfHDP-HAGlmS inducible knockdown transgenic line was generated, although our attempts to knockout PfHDP gene failed. Knock-down of HDP in the parasite line using the inducer glucosamine induced food vacuole abnormalities and parasite stress. Growth inhibition was observed in glucosamine treated parasites. A reduced level of PfHDP inside the transgenic lines also led to the reduction in the uptake of $\mathrm{Hb}$ from the parasite cytosol. These parasites also showed mis-localized or poorly expressed Plasmepsin 2 protein, a part of the hemozoin formation complex. These knocked-down parasites appeared to be in stress. The food vacuole was not properly developed in the PfHDP knocked-down parasites and hence the parasites exhibited gross morphological deformities. Attempts to study the transport of $\mathrm{Hb}$ in PfHSP101 DDDHA transgenic lines revealed that knockdown of 
one of the components of translocon complex reduced the ability of parasites to take up $\mathrm{Hb}$ from the erythrocyte cytoplasm. Hence the translocon machinery appeared to be essential for the uptake of $\mathrm{Hb}$ from the erythrocyte.

In summary, here we characterized PfHDP for its role in $\mathrm{Hb}$ uptake in addition to its previously characterized function in heme degradation. To characterize the role of PfHDP in $\mathrm{Hb}$ transport, here we generated a PfHDP-GFP transgenic line. Immunoprecipitation of highly synchronized culture of trophozoites from PfHDP-GFP line using anti-GFP antibody followed by LC-MS/MS analysis showed the association of $\mathrm{Hb}$ and PfHDP with each other and with the members of translocon complex such as Exp-2, PTEX150, PTEX88, HSP101 and $\operatorname{Trx} 2$, a complex known to export parasite proteins. Insilico analysis and invitro proteinprotein interaction techniques confirmed the interaction of PfHDP with Pfexp-2. We further showed that PfHDP is highly crucial in maintaining food vacuole and parasite health as attempts to knockdown the protein induced parasite stress. Hb uptake is severely affected in these transgenic parasites. The study thus emphasizes on the dual roles of Heme Detoxification Protein in $\mathrm{Hb}$ uptake as well as in conversion of heme to hemozoin. Looking at the multiple roles of PfHDP in the parasite life cycle, PfHDP appears to be an important target for new antimalarial discovery.

\section{Acknowledgement}

This work was financially supported by Department of Biotechnology, Government of India (BT/PR5267/MED/15/87/2012 and flagship project;BT/IC-06/003/91) from the Department of Biotechnology, Govt. of India. PM is a recipient of the J C Bose Fellowship awarded by SERB, Govt. of India, and work is supported by the grant (DST/20/015). We thank Prof Daniel E. Goldberg for providing us the PfHSP101 DDDHA transgenic parasites. We thank Dr Paul Gilson for critical review of the manuscript. We also thank the Rotary Blood Bank for providing human red blood cells for Plasmodium culture. P.G. was recipient of ICMR Cenetenary Post-Doctoral Fellowship, ICMR, Government of India. We thank Dr. Naresh Sahoo and Surabhi Dabral for their help in the SPR interaction experiments and confocal imaging, respectively. We thank the animal house facility for help with antibody generation in mice and rabbit.

\section{Ethics statement}


561 The animal work performed in this study was approved by the Institutional Animals Ethics

562 Committee of ICGEB (IAEC-ICGEB). Rotary blood bank provided human red blood cells.

\section{Author Contributions}

564 PM conceived the idea. PM and PG designed the experiments. PG performed literature 565 analysis. PG, RP, VT, SP, IK, AP, RB and SM performed experiments. RP and DG 566 performed bioinformatics analysis. AM, DG and PM supervised the study. PG, PM wrote the 567 manuscript, and all authors read and approved the manuscript.

568 
570

571

572

573

574

575

576

577

578

579

580

581

582

583

584

585

586

587

588

589

590

591

592

593

594

595

596

597

598

599

600

601

602

\section{References}

Ashong, J. O., I. P. Blench and D. C. Warhurst (1989). "The composition of haemozoin from Plasmodium falciparum." Transactions of the Royal Society of Tropical Medicine and Hygiene 83(2): 167-172.

Bahl, A., B. Brunk, R. L. Coppel, J. Crabtree, S. J. Diskin, M. J. Fraunholz, G. R. Grant, D. Gupta, R. L. Huestis, J. C. Kissinger, P. Labo, L. Li, S. K. McWeeney, A. J. Milgram, D. S. Roos, J. Schug and C. J. Stoeckert, Jr. (2002). "PlasmoDB: the Plasmodium genome resource. An integrated database providing tools for accessing, analyzing and mapping expression and sequence data (both finished and unfinished)." Nucleic Acids Res 30(1): 8790.

Beck, J. R., V. Muralidharan, A. Oksman and D. E. Goldberg (2014). "PTEX component HSP101 mediates export of diverse malaria effectors into host erythrocytes." Nature 511(7511): 592-595.

Chugh, M., V. Sundararaman, S. Kumar, V. S. Reddy, W. A. Siddiqui, K. D. Stuart and P. Malhotra (2013). "Protein complex directs hemoglobin-to-hemozoin formation in Plasmodium falciparum." Proc Natl Acad Sci U S A 110(14): 5392-5397.

Crabb, B. S., M. Rug, T.-W. Gilberger, J. K. Thompson, T. Triglia, A. G. Maier and A. F. Cowman (2004). "Transfection of the human malaria parasite Plasmodium falciparum." Methods in molecular biology (Clifton, N.J.) 270: 263-276.

Dalal, S. and M. Klemba (2007). "Roles for two aminopeptidases in vacuolar hemoglobin catabolism in Plasmodium falciparum." J Biol Chem 282(49): 35978-35987.

de Koning-Ward, T. F., P. R. Gilson, J. A. Boddey, M. Rug, B. J. Smith, A. T. Papenfuss, P. R. Sanders, R. J. Lundie, A. G. Maier, A. F. Cowman and B. S. Crabb (2009). "A newly discovered protein export machine in malaria parasites." Nature 459(7249): 945-949.

Egan, T. J. (2008). "Haemozoin formation." Molecular and biochemical parasitology 157(2): 127-136.

Egan, T. J., J. M. Combrinck, J. Egan, G. R. Hearne, H. M. Marques, S. Ntenteni, B. T. Sewell, P. J. Smith, D. Taylor, D. A. van Schalkwyk and J. C. Walden (2002). "Fate of haem iron in the malaria parasite Plasmodium falciparum." The Biochemical journal 365(Pt 2): 343-347.

Elliott, D. A., M. T. McIntosh, H. D. Hosgood, 3rd, S. Chen, G. Zhang, P. Baevova and K. A. Joiner (2008). "Four distinct pathways of hemoglobin uptake in the malaria parasite Plasmodium falciparum." Proc Natl Acad Sci U S A 105(7): 2463-2468. 
603

604

605

606

607

608

609

610

611

612

613

614

615

616

617

618

619

620

621

622

623

624

625

626

627

628

629

630

631

632

633

634

635

636

Francis, S. E., R. Banerjee and D. E. Goldberg (1997). "Biosynthesis and maturation of the malaria aspartic hemoglobinases plasmepsins I and II." J Biol Chem 272(23): 14961-14968.

Garten, M., A. S. Nasamu, J. C. Niles, J. Zimmerberg, D. E. Goldberg and J. R. Beck (2018). "EXP2 is a nutrient-permeable channel in the vacuolar membrane of Plasmodium and is essential for protein export via PTEX." Nat Microbiol 3(10): 1090-1098.

Gupta, P., S. Mehrotra, A. Sharma, M. Chugh, R. Pandey, A. Kaushik, S. Khurana, N. Srivastava, T. Srivastava, A. Deshmukh, A. Panda, P. Aggarwal, N. S. Bhavesh, R. K. Bhatnagar, A. Mohmmed, D. Gupta and P. Malhotra (2017). "Exploring Heme and Hemoglobin Binding Regions of Plasmodium Heme Detoxification Protein for New Antimalarial Discovery." J Med Chem 60(20): 8298-8308.

Ho, C. M., J. R. Beck, M. Lai, Y. Cui, D. E. Goldberg, P. F. Egea and Z. H. Zhou (2018). "Malaria parasite translocon structure and mechanism of effector export." Nature 561(7721): 70-75.

Jani, D., R. Nagarkatti, W. Beatty, R. Angel, C. Slebodnick, J. Andersen, S. Kumar and D. Rathore (2008). "HDP-a novel heme detoxification protein from the malaria parasite." PLoS pathogens 4(4): e1000053.

Laskowski, R. A. and M. B. Swindells (2011). "LigPlot+: multiple ligand-protein interaction diagrams for drug discovery." J Chem Inf Model 51(10): 2778-2786.

Lazarus, M. D., T. G. Schneider and T. F. Taraschi (2008). "A new model for hemoglobin ingestion and transport by the human malaria parasite Plasmodium falciparum." J Cell Sci 121(11): 1937-1949.

Liu, J., I. Y. Gluzman, M. E. Drew and D. E. Goldberg (2005). "The role of Plasmodium falciparum food vacuole plasmepsins." The Journal of biological chemistry 280(2): 14321437.

Luker, K. E., S. E. Francis, I. Y. Gluzman and D. E. Goldberg (1996). "Kinetic analysis of plasmepsins I and II aspartic proteases of the Plasmodium falciparum digestive vacuole." $\mathrm{Mol}$ Biochem Parasitol 79(1): 71-78.

Mashiach, E., D. Schneidman-Duhovny, N. Andrusier, R. Nussinov and H. J. Wolfson (2008). "FireDock: a web server for fast interaction refinement in molecular docking." Nucleic Acids Res 36(Web Server issue): W229-232.

Organization, W. H. (2019). World malaria report 2019. Geneva, World Health Organization. Paul, G., A. Deshmukh, I. Kaur, S. Rathore, S. Dabral, A. Panda, S. K. Singh, A. Mohmmed, M. Theisen and P. Malhotra (2017). "A novel Pfs38 protein complex on the surface of Plasmodium falciparum blood-stage merozoites." Malaria Journal 16(1). 
637 Rathore, D., T. F. McCutchan, M. Sullivan and S. Kumar (2005). "Antimalarial drugs: 638 current status and new developments." Expert opinion on investigational drugs 14(7): 871639883.

640 Sanders, P. R., B. K. Dickerman, S. C. Charnaud, P. A. Ramsland, B. S. Crabb and P. R. 641 Gilson (2019). "The N-terminus of EXP2 forms the membrane-associated pore of the protein 642 exporting translocon PTEX in Plasmodium falciparum." J Biochem 165(3): 239-248.

643 Schneidman-Duhovny, D., Y. Inbar, R. Nussinov and H. J. Wolfson (2005). "PatchDock and 644 SymmDock: servers for rigid and symmetric docking." Nucleic Acids Res 33(Web Server 645 issue): W363-367.

646 Singh, N., P. S. Sijwali, K. C. Pandey and P. J. Rosenthal (2006). "Plasmodium falciparum: 647 biochemical characterization of the cysteine protease falcipain-2'." Exp Parasitol 112(3): 187648192.

649 Tina, K. G., R. Bhadra and N. Srinivasan (2007). "PIC: Protein Interactions Calculator." $650 \quad$ Nucleic Acids Res 35(Web Server issue): W473-476.

651 Trager, W. and J. B. Jensen (1976). "Human malaria parasites in continuous culture." Science 652 (New York, N.Y.) 193(4254): 673-675.

653 Tsumoto, K., D. Ejima, I. Kumagai and T. Arakawa (2003). "Practical considerations in 654 refolding proteins from inclusion bodies." Protein Expression and Purification 28(1): 1-8.

655 Woodrow, C. J. and N. J. White (2017). "The clinical impact of artemisinin resistance in 656 Southeast Asia and the potential for future spread." FEMS microbiology reviews 41(1): 3465748.

658 Wu, Y., Q. Li and X.-Z. Chen (2007). "Detecting protein-protein interactions by Far western 659 blotting." Nature protocols 2(12): 3278-3284.

660 


\section{Figures}

A
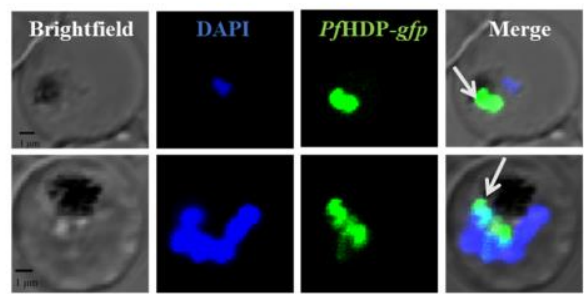

B

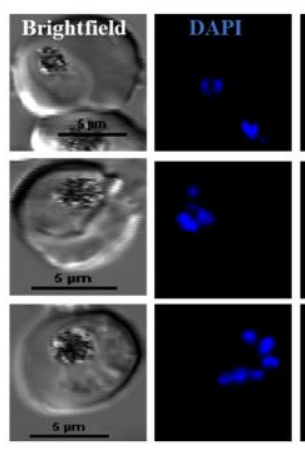

C

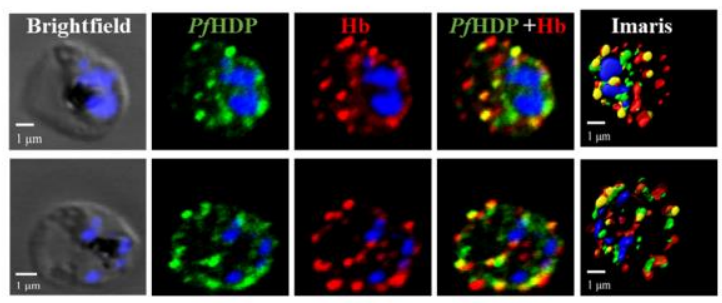

D

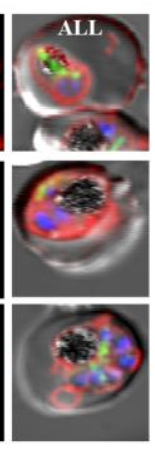

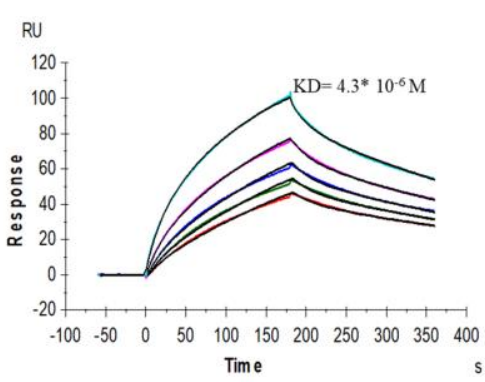

663

664 Fig. 1. A. Subcellular localization of PfHDP in PfHDP-GFP transgenic lines. The image 665 shows PfHDP is trafficked to the vesicles of the parasite B. The BODIPY-TR ceramide stain, 666 which stains the lipid membranes shows PfHDP-GFP are trafficked from vesicles to the 667 parasite plasma membrane (C) PfHDP colocalizes with $\mathrm{Hb}$, both inside the parasite as well as 668 in the cytoplasm of erythrocyte (pearson coefficient co-relation - 0.8) (D) PfHDP interacts 669 with $\mathrm{Hb}$ in an SPR experiment. The interaction is a two-state reaction, and the observed 670 dissociation constant is $4.3^{*} 10^{-6} \mathrm{M}$ for the reaction. 


\section{A}
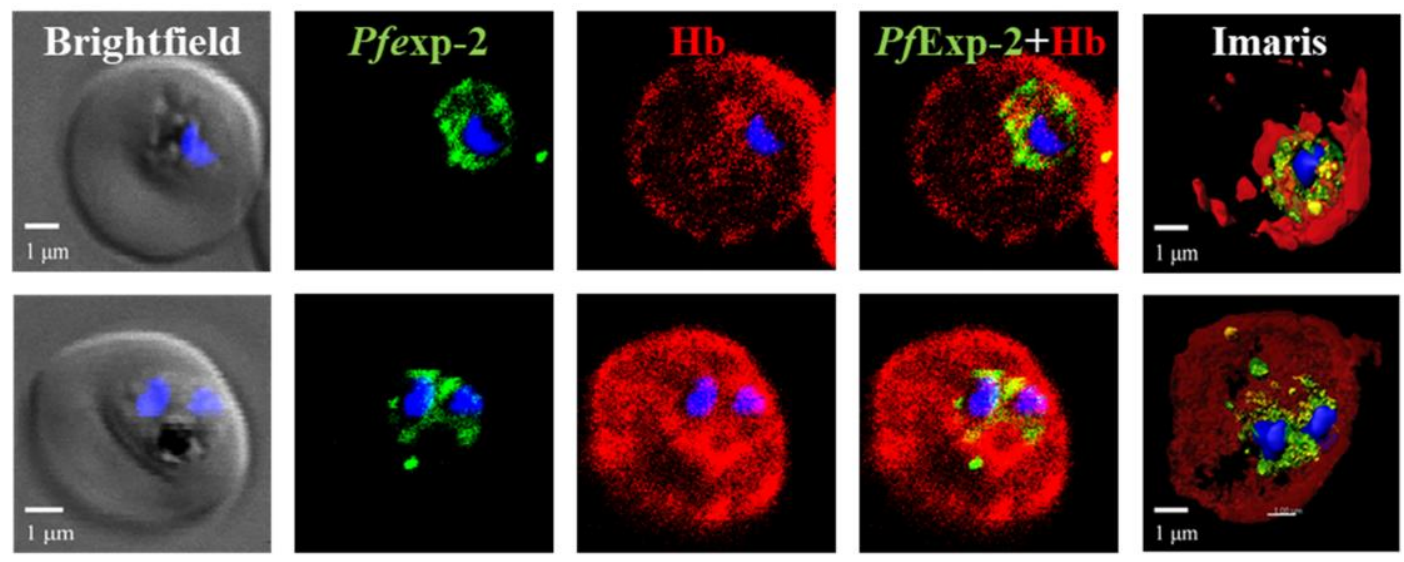

\section{B}
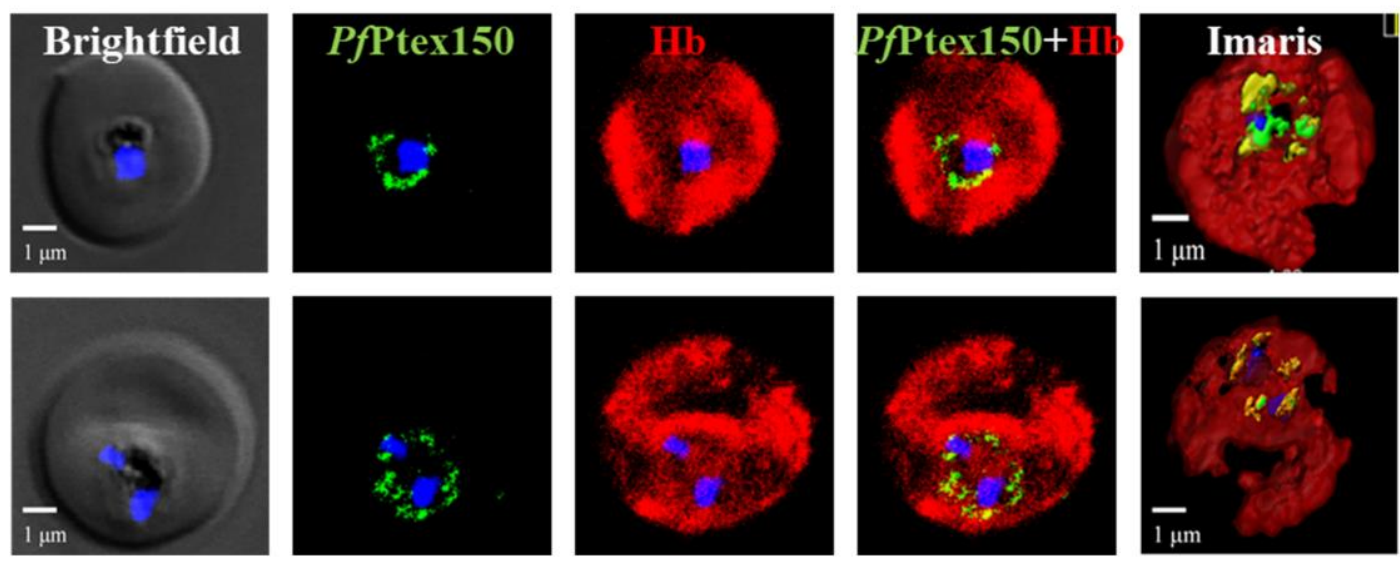

671

672 Fig. 2. Hb colocalized with members of translocon components, Pfexp-2 (A) and PfPTEX150

673 (B) with a pearson coefficient of 0.62 and 0.64 , respectively. 
A
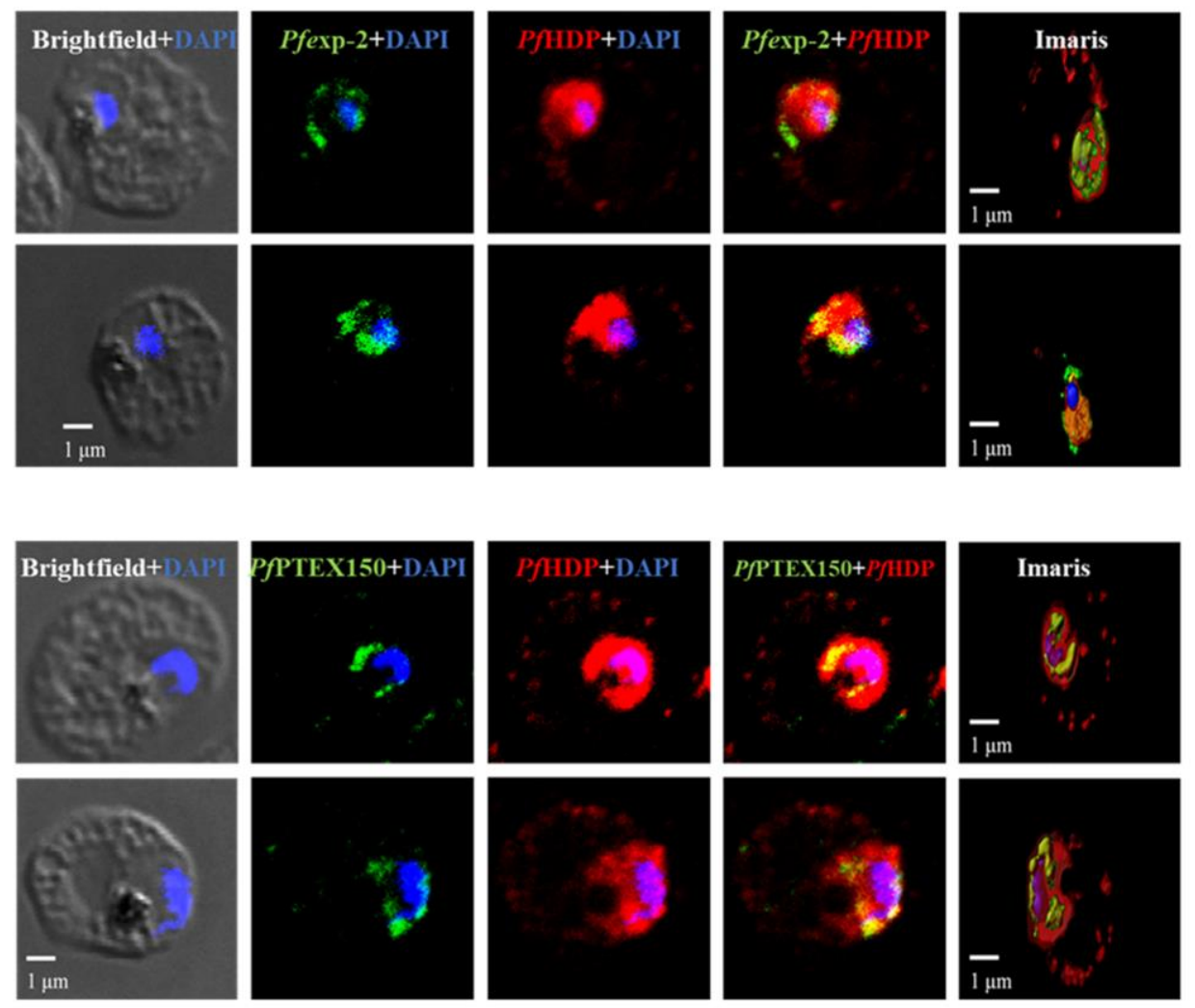

B

MBP $\quad P f H D P \quad \mathrm{M} \quad(\mathrm{kDa})$

C
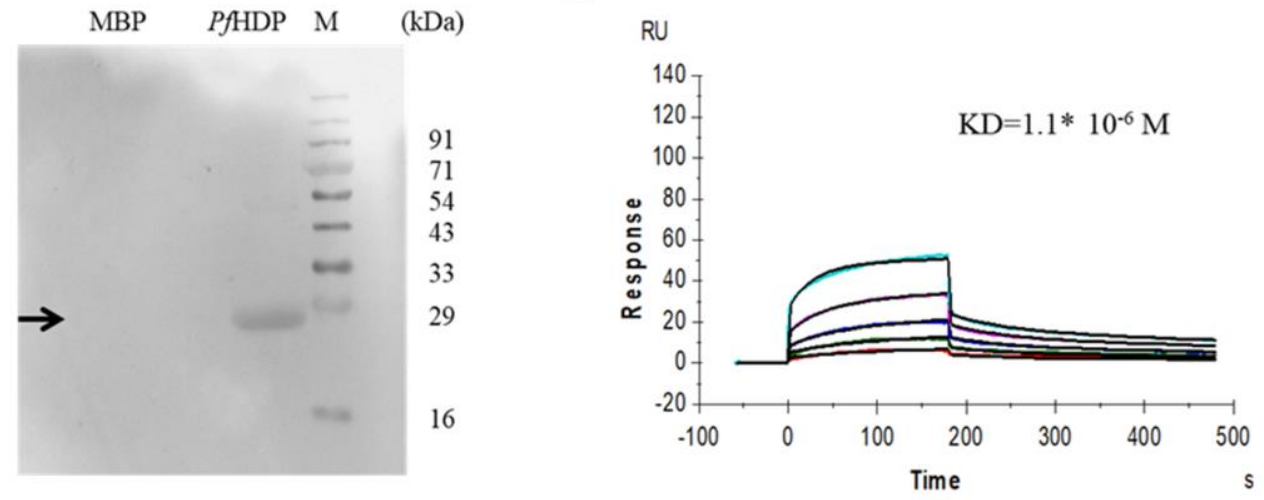

D

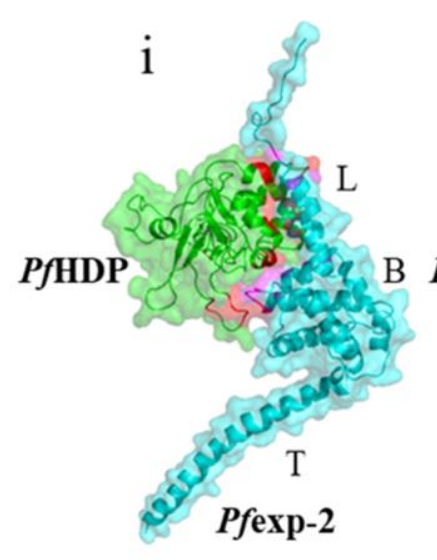

ii

iii
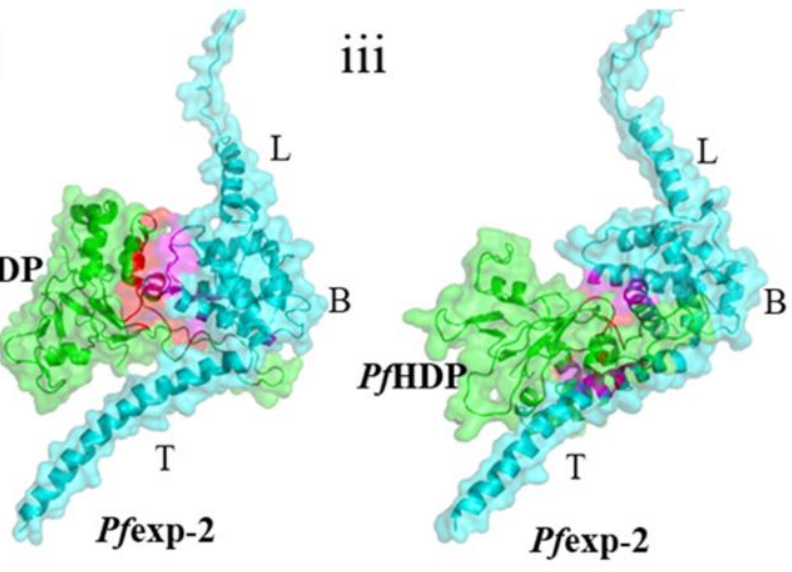
675 Fig. 3. (A) PfHDP colocalizes with the members of translocon components; Pfexp-2 and 676 PfPTEX150 at the parasitophorous vacuolar membrane with a pearson coefficient of 0.74 and 677 0.60, respectively. (B) Pfexp-2 interacts with PfHDP in a far western experiment (C) PfHDP 678 interacts with recombinant C-terminal Pfexp-2 in an SPR experiment. The interaction is a 679 two-state reaction, and the dissociation constant is 1.1* 10-6 M. (D) Conformational docking 680 patterns (i-iii) observed for The Pfexp-2 (monomer)-PfHDP complex. Green-PfHDP, Cyan681 Pfexp-2, Red - Pfexp-2 interacting region within $4 \AA$ of PfHDP, Magenta - PfHDP interacting 682 region within $4 \AA$ of $P f \exp -2$.

A

B
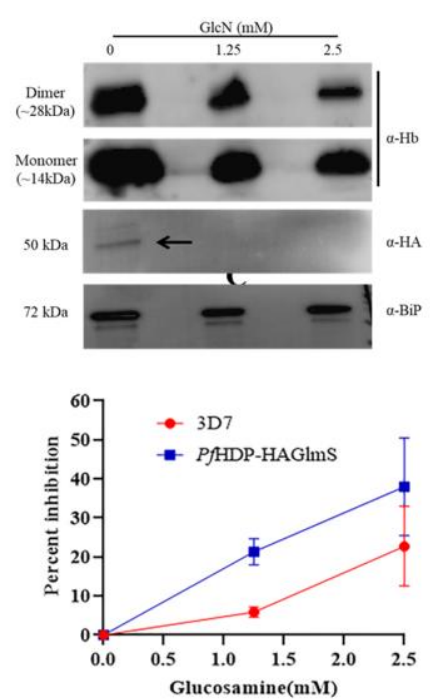

D
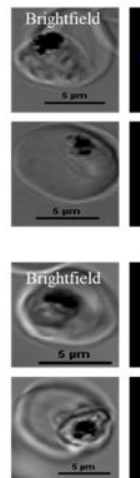
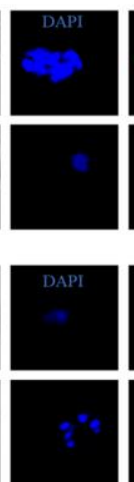
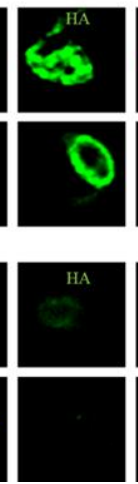

C
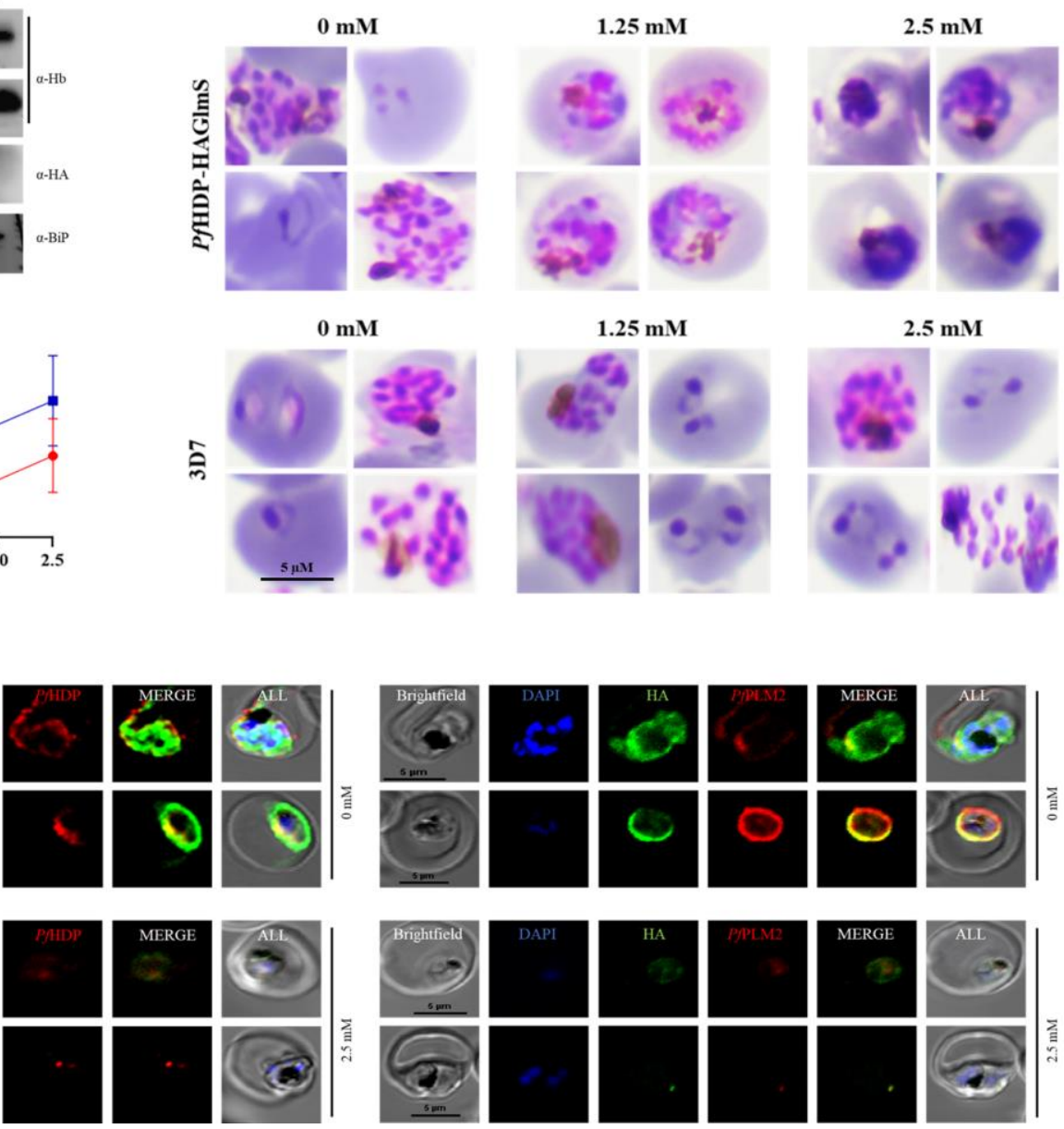

684

Fig. 4. (A) Effect of conditional knockdown of PfHDP in parasites showing up to $45 \%$ invasion inhibition. Data represents mean $\pm \mathrm{SD} ; \mathrm{n}=3$ experiments. (B) Western blot analysis of lysate from PfHDP-HA-glmS line with $\alpha$-HA rat serum and $\alpha-\mathrm{Hb}$ antibody. $\mathrm{Hb}$ uptake is reduced in the knockdown parasites. Anti BiP was used as loading control. (C) 
689 following PfHDP knockdown. (D) Immunofluorescence assays show the low levels of $690 \quad$ PfHDP and plasmepsin-2 in knockdown lines.

691

692

A

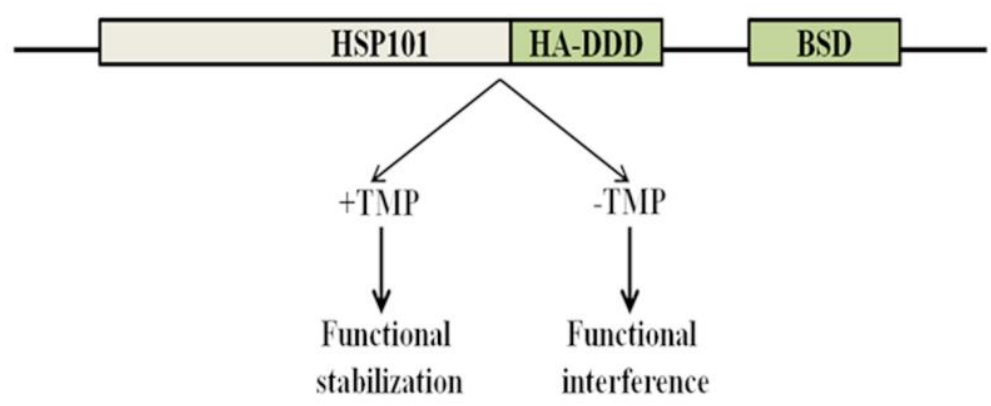

B

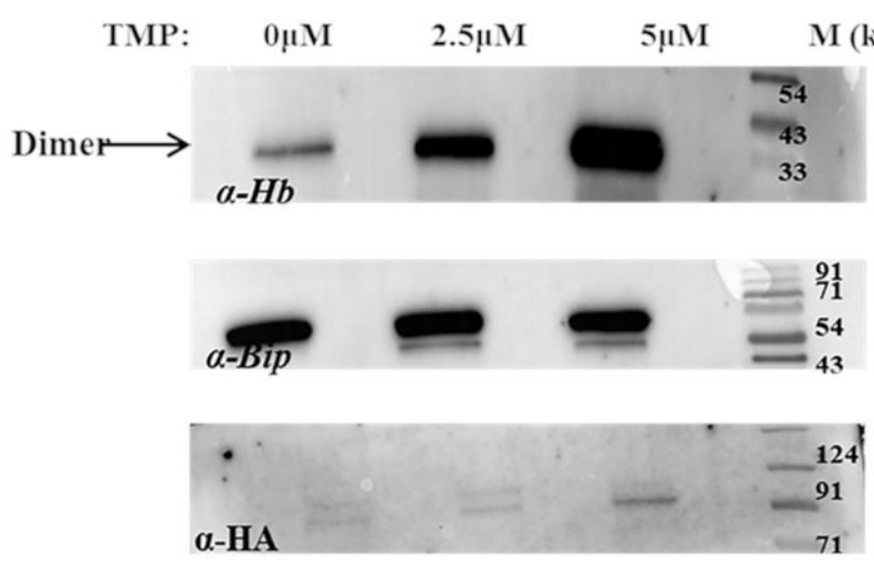

693 Fig. 5. HSP101 DDDHA knockdown parasites take up less Hb from the host erythrocyte 694 cytoplasm. (A) Illustration of the HSP101 DDDHA transgenic construct (B) western blot to 695 detect the $\mathrm{Hb}$ levels inside the parasite in HSP101 knockdown parasites. BiP is used as a 696 loading control. 
A

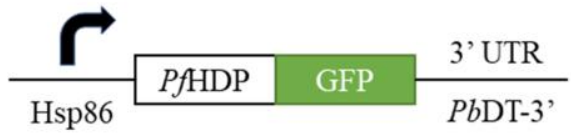

C

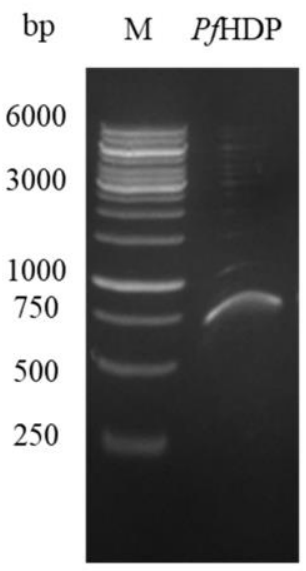

$\mathbf{E}$

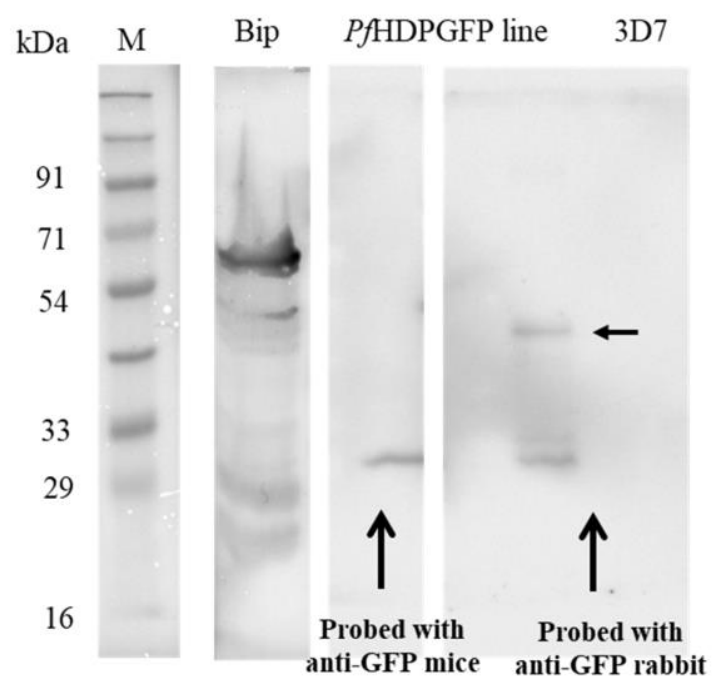

B

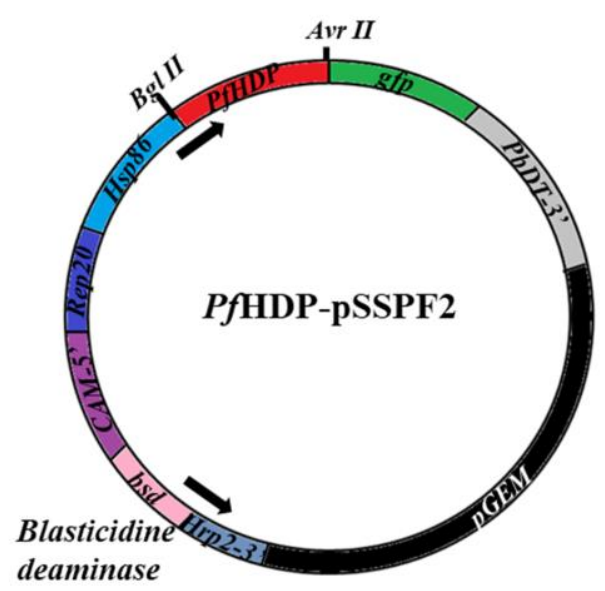

D

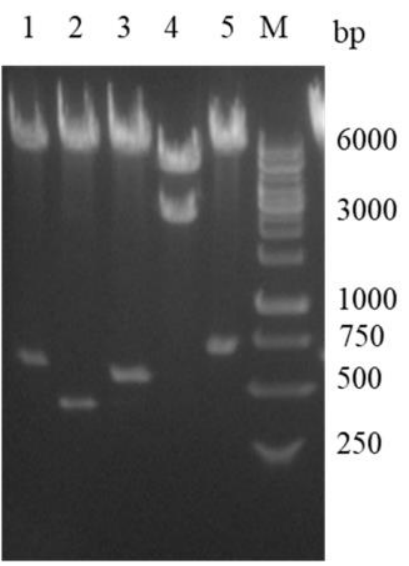

F

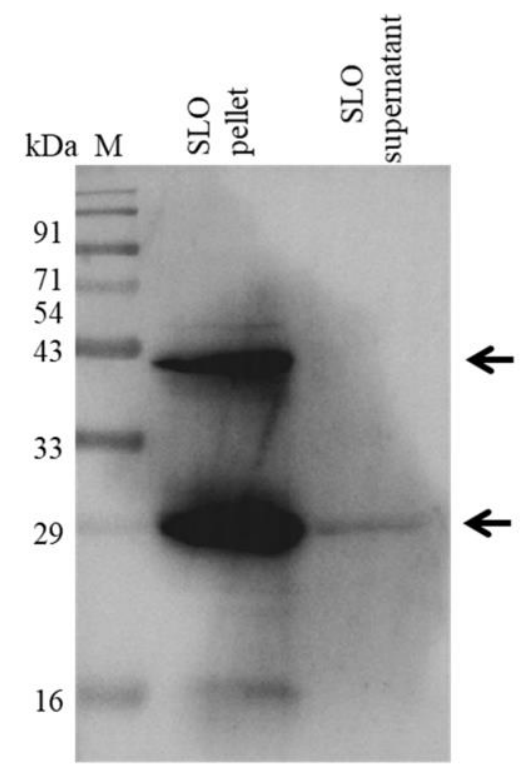


700

701

702

703

704

705

706

707

708

709

710

Sup fig. 1. Generation of PfHDP-GFP line in pSSPF2 vector. (A) Full length PfHDP gene cloned in pSSPF2 vector with GFP tag at 3' end under the control of HSP86 promoter (B) plasmid vector pSSPF2 for expressing gene products in malarial transfectants (C) PCR amplification of $P f H D P$ gene from cDNA using the PfHDP-GFP FP and PfHDP-GFP RP primer set. (D) The construct PfHDP-pSSPF2 was checked for correct insertion of PfHDP and presence of other sequence regions using different sets of restriction enzymes (Lane 1: BgIII/AvrII-HDP; Lane 2: BamHI/HindIII- blasticidine resistance; Lane3: EcoRI/HindIIIHRP 2-3', Lane 4: NotI/EcoRI- pGEM backbone, Lane 5: XhoI/AvrII: GFP (E) Western blot to confirm the expression of GFP fusion protein in the PfHDP-GFP transgenic line. PfBiP was used as a positive control. (F) PfHDPGFP detection in the SLO pellet and supernatant fractions suggesting PfHDP is transported to the erythrocyte cytosol.

A

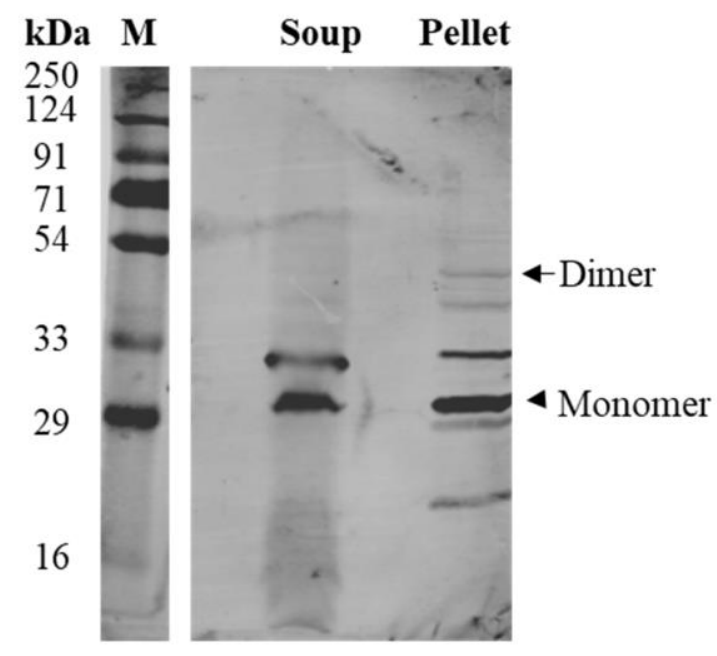

B

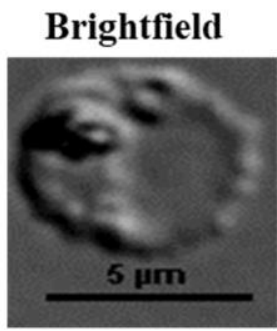

DAPI

PfHDP
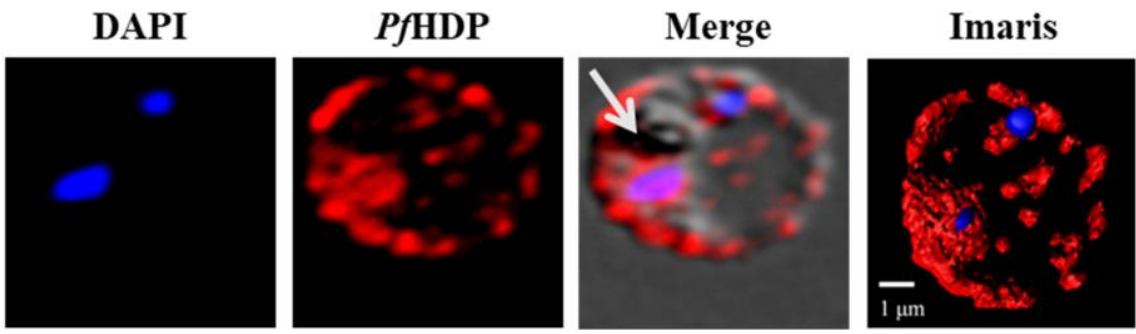

712 Sup fig. 2. (A) PfHDP is detected in both the fractions of SLO treated infected erythrocytes.

The protein is detected in both the parasite cytoplasm and erythrocyte cytoplasm by PfHDP 

$P f H D P($ red) in both the erythrocyte cytosol and parasite cytoplasm.

A

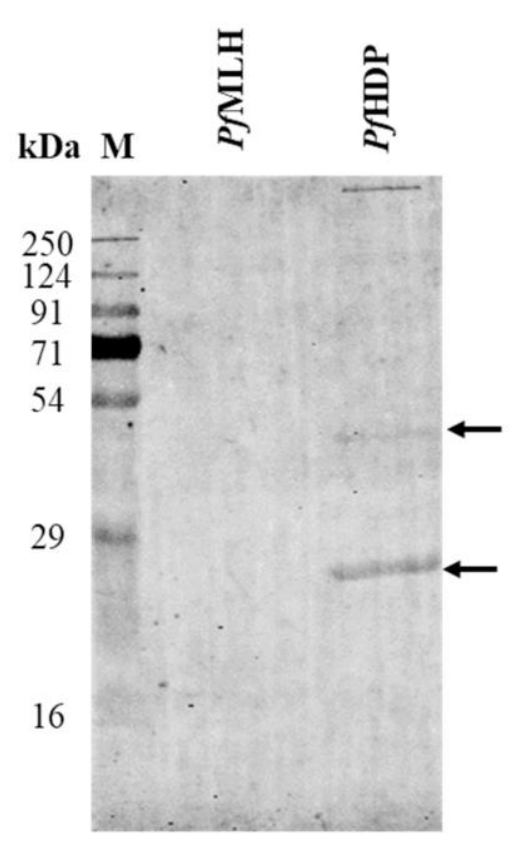

B

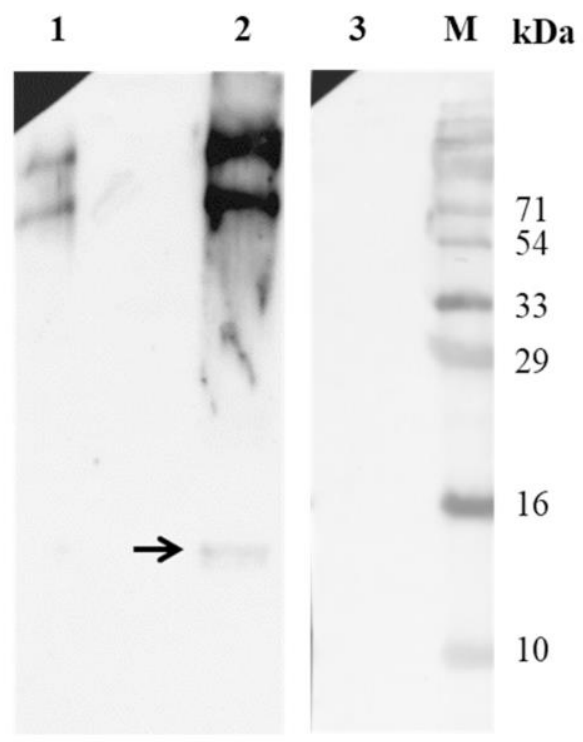

718 Sup fig. 3. (A) PfHDP interacts with $\mathrm{Hb}$ in a far western interaction experiment. Here, 719 PfMLH was used as a negative control. (B) PfHDP and $\mathrm{Hb}$ interact with each other in a co720 immunoprecipitation experiment. Lane 1 contains elute pulled from the mixture of PfHDP 721 and $\mathrm{Hb}$ using Pre immune sera. Lane 2 contains elutes pulled from a mixture of PfHDP and $722 \mathrm{Hb}$ using the HDP antibody. Lane 3 contains an eluted mixture of HDP and ClpQ using the PfHDP antibody. The arrow shows $\mathrm{Hb}$ pulled by $P f H D P$ antibody as probed by $\mathrm{Hb}$ antibody. 
A

\begin{tabular}{l|l|lll|}
\hline \multicolumn{1}{l|}{} & & Expressed region & \\
\hline 1 & 20 & 139 & 285
\end{tabular}

B

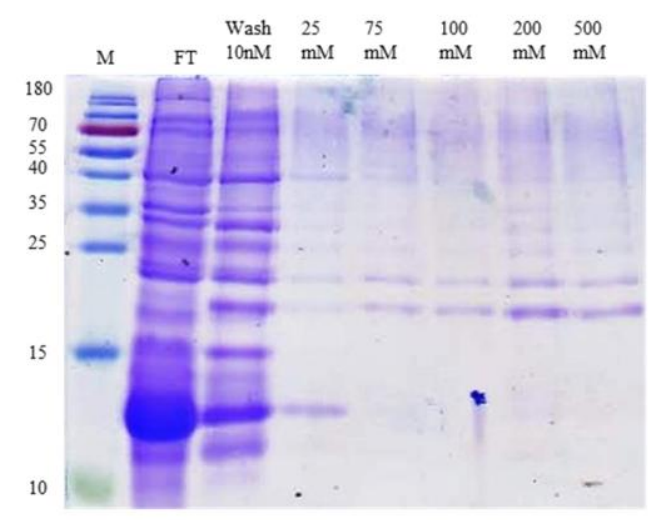

C

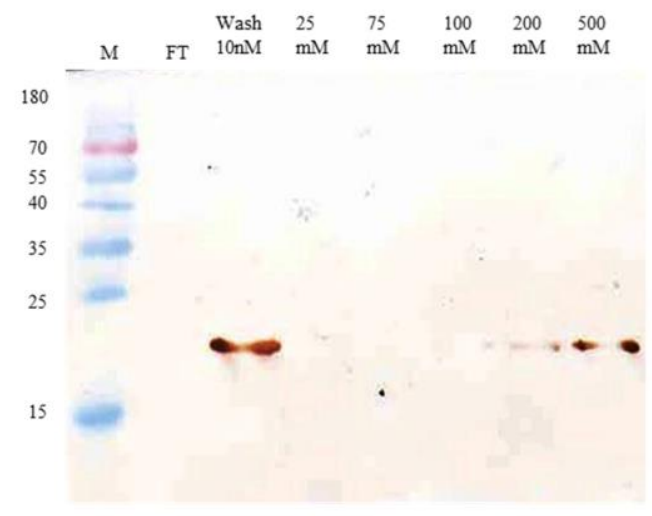

D

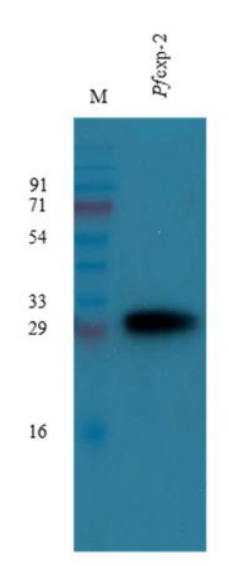

$\mathbf{E}$

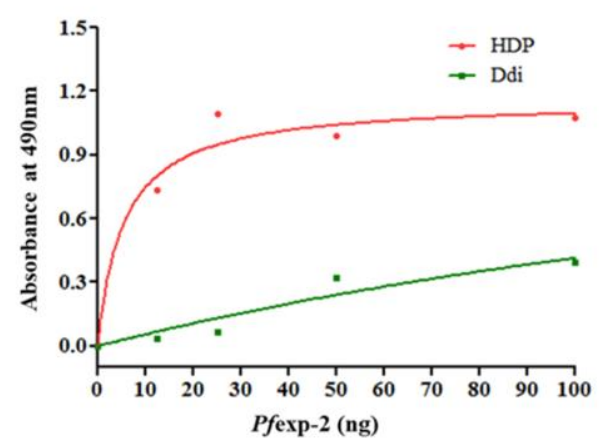

$\mathbf{F}$

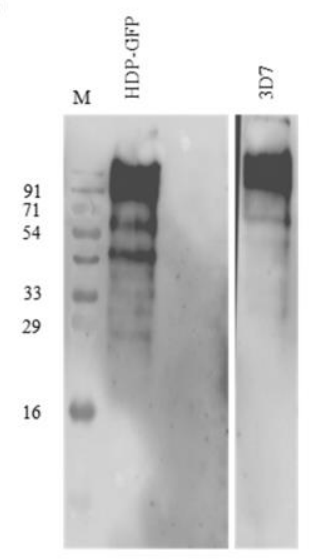

725 Sup fig. 4. (A) Pfexp-2 - C-terminal from amino acids 139-285 was expressed in pET28b vector in E. coli. (B) Coomassie stained SDS-PAGE of different elutes of recombinant Pfexp-

7272 using urea purification strategy (C) Western blot of different fractions of recombinant purified Pfexp-2 using His-HRP antibody (D) Antibody generated in rabbit against the recombinant $P f$ exp-2 protein recognized the monomeric native protein in 3D7 parasite lysate (E) Recombinant Pfexp-2 interacts with PfHDP in a dose dependent manner in an ELISA experiment. Recombinant PfDdi protein was used as a negative control. (F) western blot analysis of elutes of parasite lysate of PfHDP-GFP parasites immunoprecipitated with GFP antibody using anti- exp-2 antibody detected $P f$ exp- 2 in a western blot analysis. 
$\mathbf{A}$

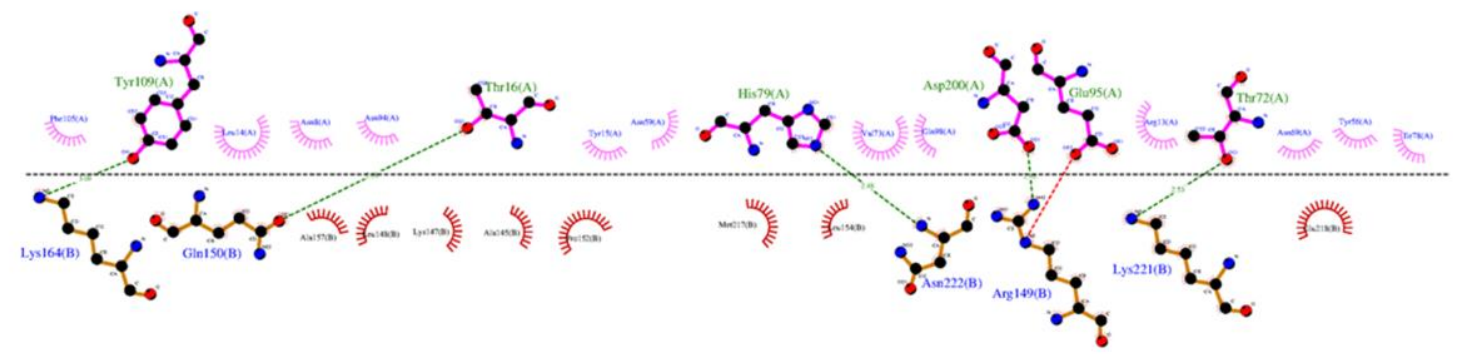

B

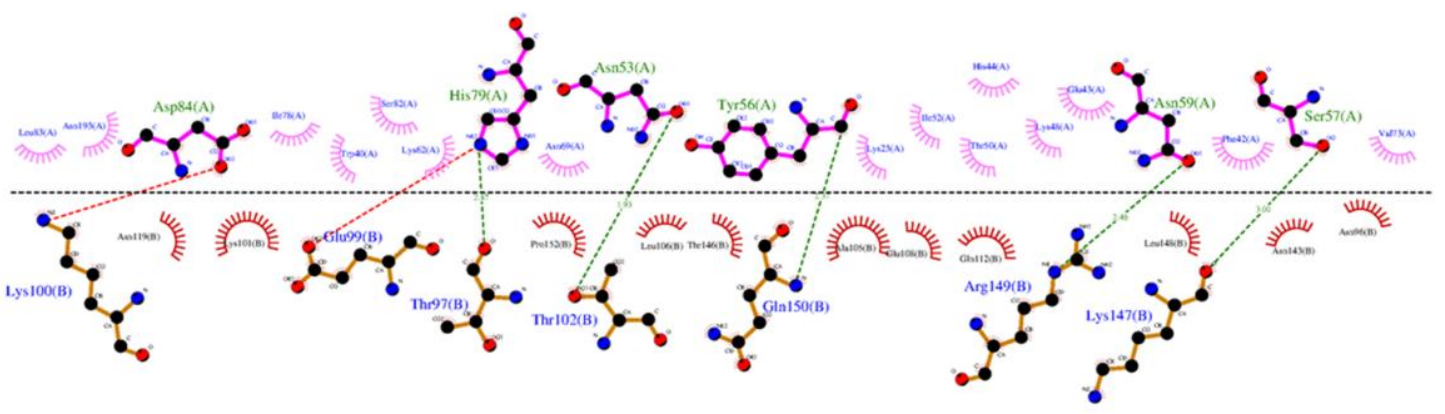

C

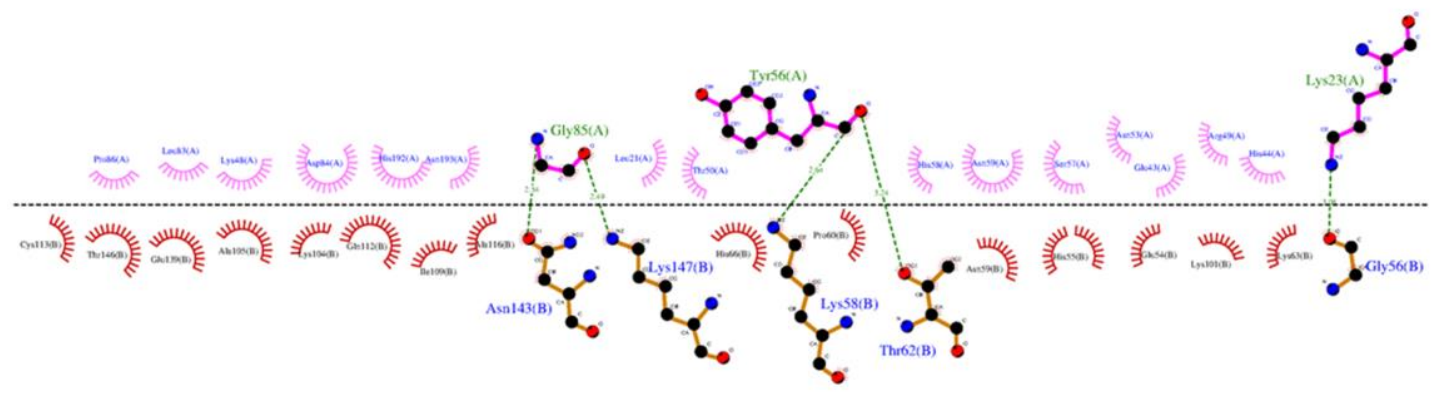

735 Sup fig. 5. DIMPLOT analysis map for $P f \exp -2$ and $P f H D P$ interacting residues in pose 1

736 (A), pose $2(B)$ and pose $3(\mathrm{C})$. 
bioRxiv preprint doi: https://doi.org/10.1101/2021 08.02.454712; this version posted August 2, 2021. The copyright holder for this preprint (which was not certified by peer review) is the author/funder, who has granted bioRxiv a license to display the preprint in perpetuity. It is made available under aCC-BY 4.0 International license.

A

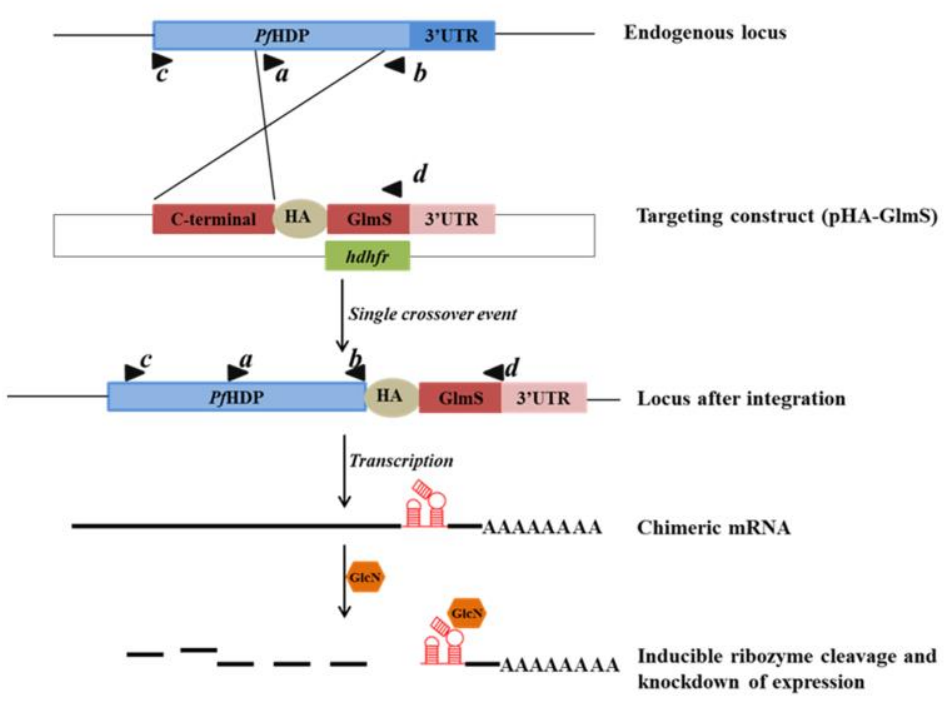

B

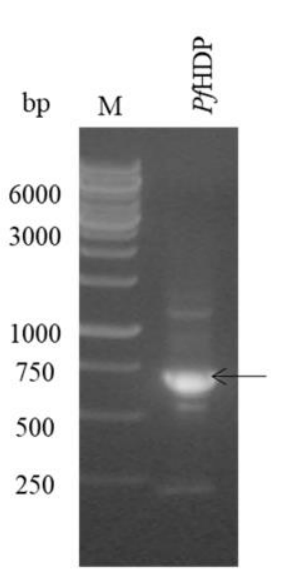

C

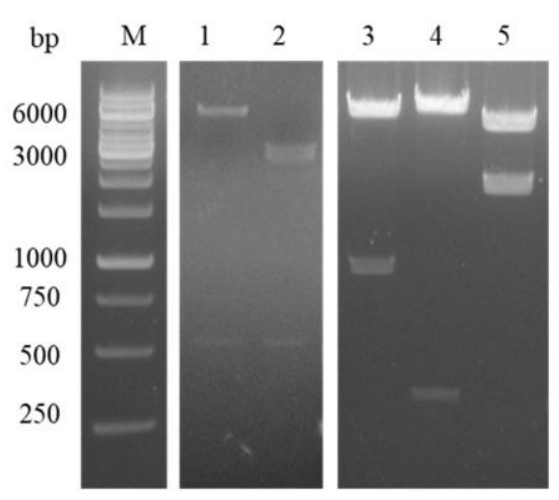

D

$\mathbf{E}$
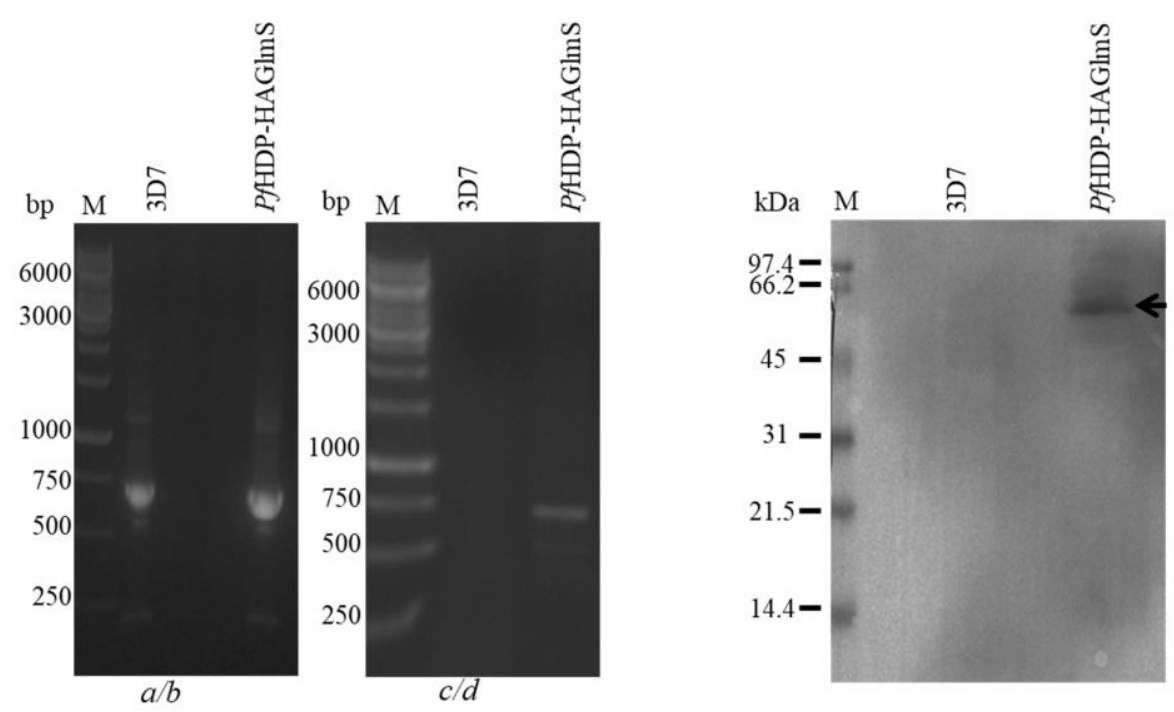
738 Sup fig. 6. Generation of PfHDP-HAGlmS line in pHA- GlmS vector. (A) Schematic of the

739 GlmS ribozyme reverse genetic tool: The ribozyme is inserted in the 3'-UTR after the coding

740 region so that it is present in the expressed mRNA. Following addition of the inducer,

741 glucosamine, which binds to the ribozyme, the mRNA self-cleaves resulting in degradation of

742 the mRNA and knock down of protein expression. (B) PCR amplification of PfHDP genomic

743 sequence from gDNA using the PfHDPGA_FP and PfHDPGA_RP primer set (C) The

744 construct PfHDP-HAglmS was checked for correct insertion of HDP and presence of other

745 sequence regions using different sets of restriction enzymes (Lane 1: BamHI/HindIII-DHFR;

746 Lane 2: EcoRI/HindIII- HRP; Lane3: BglII/PstI-PfHDP gene, Lane 4: PstI/XhoI- HA_glmS

747 ribozyme, Lane 5: XhoI/SacI: 3'-UTR (D) PCR amplification to check the integration of

748 PfHDP-HAGlmS in parasite genome. (E) Western blot of parasite lysates using anti- HA

749 antibody shows expression of HA tag fused to the PfHDP genomic locus. 
751 Table 1. List of proteins pulled down by GFP- Trap beads from lysates of PfHDP-GFP

752 parasites from P. falciparum.

\begin{tabular}{|c|l|r|r|}
\hline Gene ID & \multicolumn{1}{|c|}{ Protein } & MW (kDa) & Peptides \\
\hline PF3D7_1446800 & Heme Detoxification Protein (HDP) & 24.3 & 3 \\
\hline PF3D7_1471100 & Exported protein 2 (EXP-2) & 33.4 & 8 \\
\hline PF3D7_1345100 & Thioredoxin 2 (TRX2) & 18.6 & 1 \\
\hline PF3D7_1105600 & Translocon component PTEX88 (PTEX88) & 90.7 & 1 \\
\hline PF3D7_1116800 & Heat shock protein 101 (HSP101) & 102.8 & 4 \\
\hline
\end{tabular}

753

754 Table 2. List of proteins pulled down by anti-Hb antibody from lysates of $P$. falciparum

755 parasites.

\begin{tabular}{|c|l|r|r|}
\hline Gene ID & \multicolumn{1}{|c|}{ Protein } & MW (kDa) & Peptides \\
\hline PF3D7_1436300 & Translocon component PTEX150 (PTEX150) & 25.74 & 7 \\
\hline PF3D7_1105600 & Translocon component PTEX88 (PTEX88) & 90.7 & 1 \\
\hline PF3D7_1471100 & Exported protein 2 (EXP-2) & 33.4 & 5 \\
\hline PF3D7_1116800 & Heat shock protein 101 (HSP101) & 102.8 & \\
\hline PF3D7_1345100 & Thioredoxin-2 (TRX-2) & 18.6 & 5 \\
\hline
\end{tabular}

756

757 Supplementary Table 1. The list of Pfexp-2 and PfHDP interacting residues involved in 758 protein-protein interaction. 